\title{
Effect of Moisture Regimes, FYM and Levels of P Carriers on Phosphorus Fractions Status of Loamy Sand in Laboratory Condition
}

\author{
Kashyap N. Patel ${ }^{\text {** }}$, D. A. Patel ${ }^{1}$, Vidhi K. Patel ${ }^{1}$, Foram B. Patel ${ }^{2}$, \\ V. R. Patel ${ }^{1}$ and R. P. Pavaya ${ }^{1}$
}

${ }^{1}$ Department of Agricultural Chemistry and Soil Science, C. P. College of Agriculture, Sardarkrushinagar Dantiwada Agricultural University, Sardarkrushinagar - 385 506, Gujarat, India

${ }^{2}$ Centre for Natural Resources Management, Sardarkrushinagar Dantiwada Agricultural University, Sardarkrushinagar - 385 506, Gujarat, India

*Corresponding author

\section{A B S T R A C T}

\begin{tabular}{|l|}
\hline Ke y w o r d s \\
Moisture regimes, \\
Levels of \\
phosphorus, \\
Phosphorus carriers, \\
Incubation \\
intervals, \\
Phosphorus \\
fractions
\end{tabular}

The principle of this study was to investigate the effect of moisture regimes, FYM and levels of $\mathrm{P}$ carriers on phosphorus fractions status of loamy sand in vitro condition. Incubation study was carried out during 2017, in the Laboratory of Department of Agricultural Chemistry and Soil Science, C. P. College of Agriculture, S. D. Agricultural University, Sardarkrushinagar. Treatments comprising of three levels of moisture regimes, three levels of phosphorus of two P carriers, two levels of FYM and five incubation intervals were evaluated under a completely randomized design (with factorial concept) with three replications. Available phosphorus content in soil was increased significantly with the application of FYM (10 t/ha) with $\mathrm{P}_{2}(2.68 \mathrm{mg} \mathrm{P} / 100 \mathrm{~g}$ soil) levels of $\mathrm{P}$ and maintenance of moisture at $\mathrm{W}_{3}(25 \%$ Available water capacity) level was found significantly higher as compared to without FYM. Available P increased up to $3{ }^{\text {rd }}$ DAI then decreased concerning phosphorus availability in loamy sand. Under the different forms of phosphorus, maintenance of $\mathrm{W}_{1}(100 \%$ Available water capacity) moisture regime, FYM @ $10 \mathrm{t} / \mathrm{ha}$ and $\mathrm{P}$ level with $2.68 \mathrm{mg}$ P/100 g soil increased the in Organic-P and Total-P up to $14^{\text {th }}$ DAI therefor, scarcity of available P increased at initial stage. The concentration and contribution of each fraction to Total-P was in the order: Occluded-P < Al-P $<$ Saloid-P $<$ Reductant-P $<$ Fe-P $<$ Organic-P $<$ Ca-P.

\section{Introduction}

Phosphorus (P) is essential for plants and animals because of its role in vital life processes, such as in photosynthesis in plants and energy transformations in all forms of life. It also has a significant role in sustaining and building up soil fertility, particularly under intensive systems of agriculture. Soils are known to vary widely in their capacities to supply $\mathrm{P}$ to crops because only a small fraction of the total $\mathrm{P}$ in soil is in a form 
available to crops. Thus, unless the soil contains adequate amount of plant-available $\mathrm{P}$, or is supplied with readily available(inorganic)-P fertilizers, crop growth will suffer (Sanyal and Datta 1991).

Although $\mathrm{P}$ is one of the most important factors to limit soil ecosystem productivity (Zhu et al., 2004), plant and soil microbiota responses to $\mathrm{P}$ addition could sometimes be inconsistent (Nielsen et al., 2015) due to different soil moisture conditions the majority of soil types, even when fertile, lack phosphorus because its renewal in soil solutions takes time compared to the root uptake (Suriyagoda et al., 2011). Besides, drought could enhance phosphorus deficiency, as it is excessively immobile in soil (Sardans and Penuelas 2007). A decrease in soil water availability affects the rate of diffusion of many plant nutrients and finally the composition and concentration of soil solution. Throughout water stress a marked decrease in nutrient uptake is reported (Marschner 1986) through the decreased transfer of ions to the root. Thus, it will be of significant use to quantify the level of water stress above which the mobilization and absorption of nutrients are adversely affected.

Phosphorus availability in soils is affected by several factors such as soil reaction, organic matter, texture (Verma 2013), calcium carbonate (Hopkins and Ellsworth 2005), parent material, weathering and climatic conditions (Fuentes et al., 2008). The suitability extent of calcareous soils for agriculture depends on management systems via adding organic materials and some amendments to improve the availability of nutrients, particularly phosphorus (Al-Oud 2011; Karimi et al., 2012). Organic manure additions also caused an increment in Olsen extractable P of soil (Bahl and Toor 2002). In P-fixing soils, applications of organic matter were reported to increase available $\mathrm{P}$ because of mineralization (Iyamuremye and Dick 1996). Decomposition of FYM produces different organic acids, which help in mobilizing non-labile $\mathrm{P}$ in soil into labile $\mathrm{P}$. Phosphorus uptake is enhanced by the addition of organics due to production of organic acids which in turn, transform $\mathrm{P}$ from non-utilizable form to plant utilizable form (Ivanova et al., 2006). Thus, the incorporation of FYM improves soil health and crop yield (Dotaniya 2012).

Maintenance of an adequate amount of soil $\mathrm{P}$ through the application of inorganic and organic P is critical for the sustainability of the cropping system (Sharpley et al., 1994). For phosphorus requirement plants depend on inorganic form of phosphorus. It has now been established that Saloid-P, Aluminium-P (Al-P), Iron-P (Fe-P), and Calcium-P (Ca-P) are the major soil inorganic fractions and their relative proportion depends upon various factors (Jaggi 1991). The availability and fractions of soil $\mathrm{P}$ may change due to longterm continuous $\mathrm{P}$ fertilization besides its yield-increasing effect (Fan et al., 2003; Lai et al., 2003). Therefore, the present investigation has been framed with the objectives of studying the effect of moisture regimes, FYM and levels of $\mathrm{P}$ carriers on phosphorus fractions status of loamy sand in vitro condition.

\section{Materials and Methods}

\section{Initial Physico-chemical properties of the soil}

The representative soil sample was analyzed for different Physico-chemical characteristics. The soil of the experimental site was loamy sand in texture. The soil was low in organic carbon $(0.23 \%)$ and available nitrogen (209.36 kg/ha), medium in available $\mathrm{P}_{2} \mathrm{O}_{5}$ $(33.15 \mathrm{~kg} / \mathrm{ha})$ and $\mathrm{K}_{2} \mathrm{O} \quad(231.78 \mathrm{~kg} / \mathrm{ha})$ whereas, EC (0.18 dS/m at $\left.25^{\circ} \mathrm{C}\right), \mathrm{pH}(7.50)$ 
at $25{ }^{\circ} \mathrm{C}$, bulk density $\left(1.65 \mathrm{Mg} / \mathrm{m}^{3}\right)$ and Maximum water holding capacity (22.12\%).

\section{Experimental details of incubation study}

Incubation study was carried out in the Department of Agricultural Chemistry and Soil Science, Chimanbhai Patel College of Agriculture, Sardarkrushinagar Dantiwada Agricultural University, Sardarkrushinagar during, 2017. Five hundred gram of soil was taken and required quantity of FYM was added as per treatment followed by a solution of $\mathrm{P}$ representing each source was added in each set of respective treatment to give the desired concentration of P. The sample was then transferred to $1000 \mathrm{ml}$ capacity plastic beaker and the desired moisture regime was brought. After adjustment of the moisture regime, the weight of each beaker was recorded for maintaining the moisture throughout the incubation period. The moisture was maintained by adding the amount of water every day equivalent to the loss in weight. A known amount of sample was withdrawn from each treatment at stipulated intervals. Simultaneously, the sample was also withdrawn for the determination of moisture. The sample was taken as per the interval for the determination of available $\mathrm{P}_{2} \mathrm{O}_{5}$ and $\mathrm{P}$ fractions content in soil. The Total-P was determined by digesting $1.0 \mathrm{~g}$ of $0.15 \mathrm{~mm}$ sieved oven-dried soil with $\mathrm{HNO}_{3}$ and $\mathrm{HClO}_{4}$ acids and then followed the vanadomolybdate method (Hesse 1971). The Inorganic-P was extracted with concentrated $\mathrm{HCl}$ (Hesse 1971), and the P in solution was determined with cholorotanuous reduced molybdophosphoric blue color method in $\mathrm{HCl}$ system (Jackson 1978). The difference between total and Inorganic-P was reported as Organic-P. The fractions of the Inorganic-P, which includes Saloid bound-P, Al-P, Fe-P, Reductant soluble-P, Occluded-P and Calcium-P was extracted successively by the method of Chang and Jackson (Petersen and
Corey, 1966) and the blue color was also developed as described by them.

\section{Details of incubation study}

\begin{tabular}{|c|c|c|c|}
\hline \multicolumn{4}{|c|}{ Moisture regimes: 03} \\
\hline $\mathrm{W}_{1}$ & $=$ & $\begin{array}{l}100 \\
\text { capacit }\end{array}$ & $\begin{array}{l}\text { Available } \\
\text { AWC) }\end{array}$ \\
\hline $\mathrm{W}_{2}$ & $=$ & $\begin{array}{l}50 \quad \% \\
\text { capacit }\end{array}$ & Available \\
\hline $\mathrm{W}_{3}$ & $=$ & $\begin{array}{l}25 \quad 0 \\
\text { capacit }\end{array}$ & Available \\
\hline
\end{tabular}

Levels of P: 03

$\mathrm{P}_{0} \quad=0.00 \mathrm{mg} \mathrm{P} / 100 \mathrm{~g}$ soil

$\mathrm{P}_{1} \quad=1.34 \mathrm{mg} \mathrm{P} / 100 \mathrm{~g}$ soil

$\mathrm{P}_{2} \quad=2.68 \mathrm{mg} \mathrm{P} / 100 \mathrm{~g}$ soil

Sources of P: 02

$\mathrm{S}_{1} \quad=$ Mono-Ammonium Phosphate (MAP)

$\mathrm{S}_{2}=$ Di-Ammonium Phosphate (DAP)

FYM: 02

$\mathrm{M}_{0}=0 \mathrm{t} / \mathrm{ha}$

$\mathrm{M}_{1}=10 \mathrm{t} / \mathrm{ha}$

Incubation intervals: 05

$\begin{array}{ll}\mathrm{I}_{0} & =01^{\text {st }} \text { day } \\ \mathrm{I}_{1} & =03^{\text {rd }} \text { day } \\ \mathrm{I}_{2} & =05^{\text {th }} \text { day } \\ \mathrm{I}_{3} & =07^{\text {th }} \text { day } \\ \mathrm{I}_{4} & =14^{\text {th }} \text { day }\end{array}$

Design

CRD

(With

Factorial

Concept)

Number of repetitions : 03

Number of treatment : 36

combinations

Total number of : 540

experimental beakers

or units

\section{Results and Discussion}

Phosphorus is one of the major nutrient elements that are required in a large amount by crop plants. Because of its high requirement, it has to be added to the soil. 
However, on entering the soil, it enters into a complex cycle of fixation due to its high reactivity with various ions particularly the $\mathrm{Ca}, \mathrm{Fe}, \mathrm{Al}$, and several organic compounds. The combination of $\mathrm{P}$ with different ions and also with different organic compounds or the fixation is affected by the type of soil and its chemical composition, regimes of moisture, rate of addition of $\mathrm{P}$ and its sources through which, it is added and several other factors. Keeping the above-mentioned facts in view a laboratory incubation experiment was conducted under controlled conditions to study the effect of different moisture regimes, with and without the addition of FYM and levels of $\mathrm{P}$ carriers on the transformation of phosphorus detected in the soil as per the procedure depicted in materials and methods.

\section{Available $\mathbf{P}_{\mathbf{2}} \mathrm{O}_{5}$}

Data about the individual effect of available phosphorus $\left(\mathrm{kg} \mathrm{P}_{2} \mathrm{O}_{5} / \mathrm{ha}\right)$ content in the soil as influenced by different factors like incubation period, FYM, moisture regimes and levels of $\mathrm{P}$ carriers are presented in Fig. 1. The soil moisture regimes exerted a significant effect on the availability of $\mathrm{P}_{2} \mathrm{O}_{5}$ in soil. The behavior of available $\mathrm{P}_{2} \mathrm{O}_{5}$ in soil was in the order of $\mathrm{W}_{1}<\mathrm{W}_{2}<\mathrm{W}_{3}$ (Fig. 1(a)). The available $\mathrm{P}_{2} \mathrm{O}_{5}$ content was significantly increased from $30.99 \mathrm{~kg} / \mathrm{ha}$ under $\mathrm{W}_{1}$ to 42.87 $\mathrm{kg} / \mathrm{ha}$ under $\mathrm{W}_{3}$, on the $1^{\text {st }}$ Day After Incubation (DAI) with different moisture levels $\left(\mathrm{W}_{1}=31.30, \mathrm{~W}_{2}=35.82\right.$ and $\mathrm{W}_{3}=$ $36.55 \mathrm{~kg} \quad \mathrm{P}_{2} \mathrm{O}_{5} /$ ha) $\quad \mathrm{P}_{2} \mathrm{O}_{5}$ availability was significantly $(P<0.05)$ higher as compared to $7^{\text {th }}$ and $14^{\text {th }}$ DAI, whereas, $3^{\text {rd }}\left(\mathrm{W}_{1}=34.00\right.$, $\mathrm{W}_{2}=46.47$ and $\mathrm{W}_{3}=49.67 \mathrm{~kg} \mathrm{P}_{2} \mathrm{O}_{5} /$ ha) and $5^{\text {th }}\left(\mathrm{W}_{1}=31.82, \mathrm{~W}_{2}=42.32\right.$ and $\mathrm{W}_{3}=45.13$ $\mathrm{kg} \mathrm{P}_{2} \mathrm{O}_{5} /$ ha) DAI was significantly $(P<0.05)$ higher as compared to other different DAI with different moisture levels. The addition of $\mathrm{P}$ was found to be significantly increased the content of available $\mathrm{P}_{2} \mathrm{O}_{5}$ in soil up to the $\mathrm{P}_{2}$ level (Fig. 1(b)). The results revealed that the application of $2.68 \mathrm{mg} \mathrm{P} / 100 \mathrm{~g}$ soil $\left(\mathrm{P}_{2}\right)$ gave significantly $(P<0.05)$ the highest availability of $\mathrm{P}_{2} \mathrm{O}_{5}$ (36.74, 45.56, 41.94, 40.30 and 37.24 $\left.\mathrm{kg} \mathrm{P}_{2} \mathrm{O}_{5} / \mathrm{ha}\right)$ in $1^{\text {st }}, 3^{\text {rd }}, 5^{\text {th }}, 7^{\text {th }}$ and $14^{\text {th }}$ DAI, respectively. Different sources of phosphorus did not exert a significant $(P<0.05)$ effect on $1^{\text {st }}$ to $14^{\text {th }}$ DAI, but DAP proved its superiority over MAP concerning available $\mathrm{P}_{2} \mathrm{O}_{5}$ content in the soil (Fig. 1(c)). The addition of FYM (10 t/ha) was significantly $(P<0.05)$ increased the availability of $\mathrm{P}_{2} \mathrm{O}_{5}$ in soil from 37.20 to $39.14 \mathrm{~kg} \mathrm{P}_{2} \mathrm{O}_{5} / \mathrm{ha}$ (Fig. 1(d)). The addition of FYM @ $10 \mathrm{t} / \mathrm{ha}$ at $3^{\text {rd }}$ DAI resulted in a significantly $(P<0.05)$ higher amount of available $\mathrm{P}_{2} \mathrm{O}_{5} \quad(44.40$ $\mathrm{kg} / \mathrm{ha}$ ). Although, it is surprising to know that the addition of FYM resulted in the lowest value $(35.50 \mathrm{~kg} / \mathrm{ha})$ of available $\mathrm{P}_{2} \mathrm{O}_{5}$ content in soil at $1^{\text {st }}$ DAI.

\section{Phosphorus fractions}

\section{Saloid-P (mg/kg)}

The Saloid-P refers to the water-soluble and freely exchangeable $\mathrm{P}$ of the soil. Saloid-P content decreased with the period of incubation in all treatments. Results about the contents of Saloid-P in the soil at different intervals of incubation are presented in Figure 2. The Saloid-P content of soil as influenced by moisture regimes was found to be higher on $1^{\text {st }}$ DAI, immediately after different treatments application, as compared to $3^{\text {rd }}, 5^{\text {th }}$, $7^{\text {th }}$ and $14^{\text {th }}$ DAI (Fig. 2(a)). On the $1^{\text {st }}$ to $14^{\text {th }}$ day of incubation the Saloid-P content of soil as influenced by moisture regimes followed the order $\mathrm{W}_{1}>\mathrm{W}_{2}>\mathrm{W}_{3}$. The maximum concentration of Saloid-P was recorded in the $\mathrm{P}_{2}$ level of phosphorus $(32.95 \mathrm{mg} / \mathrm{kg})$ while the treatment receiving fertilizer $\mathrm{P}_{0}$ level (control) has resulted in the lowest value of Saloid-P (27.81 mg/kg). The results indicate that as fertilizer dose increased; the status of Saloid-P was also increased corresponding at $1^{\text {st }}$ to $14^{\text {th }}$ DAI (Fig 2(b)). The addition of 
FYM@ 10 t/ha resulted from a significantly $(P<0.05)$ higher amount of saloid phosphorus $(32.03 \mathrm{mg} / \mathrm{kg}$ ) as compared to without FYM (Fig. 2(d)). Although, it is surprising to know that the addition of FYM resulted in the lowest value $(24.00 \mathrm{mg} / \mathrm{kg})$ of saloid $P$ content in soil at $14^{\text {th }} \mathrm{DAI}$.

\section{Aluminium-P (mg/kg)}

There was not much variation in the content of Al-P anions the treatments irrespective of the days of incubation (Fig. 3). The Al-P content of soil as influenced by different treatments was found to increase up to $14^{\text {th }}$ DAI. Figure 3(a) show that the Al-P content in soil was noted higher in $\mathrm{W}_{1}(28.15 \mathrm{mg} / \mathrm{kg})$ and lowest in $\mathrm{W}_{3}(23.02 \mathrm{mg} / \mathrm{kg})$. The Al-P content was higher in $\mathrm{P}_{2}(28.60 \mathrm{mg} / \mathrm{kg})$ level of fertilizer, Al-P content of these treatments was significantly $(P<0.05)$ higher than others at all the sampling dates. Phosphorus level $\mathrm{P}_{0}$ recorded significantly $(P<0.05)$ lower Al-P $(23.55 \mathrm{mg} / \mathrm{kg}$ ) content in the soil (Fig. 3(b)). The sources of phosphorus were found nonsignificant $(P<0.05)$ on Al-P at $1^{\text {st }}$ to $14^{\text {th }}$ DAI (Fig. 3(c)), but MAP proved its superiority over DAP concerning Al-P content in soil.

The data recorded on Fe-P as influenced by different moisture regimes, FYM and levels of $\mathrm{P}$ carriers and interactions effect are graphically depicted in Figure 4. Fe-P was increased significantly $(P<0.05)$ after $1^{\text {st }}$ day to $14^{\text {th }}$ DAI. The significantly $(P<0.05)$ higher Fe-P $(68.55 \mathrm{mg} / \mathrm{kg})$ content was recorded in $\mathrm{W}_{1}$. While the minimum value of $\mathrm{Fe}-\mathrm{P}(60.75$ $\mathrm{mg} / \mathrm{kg}$ ) was recorded under $25 \%$ available water capacity (Fig. 4(a)). Higher Fe-P was recorded in the $\mathrm{P}_{2}$ level of $\mathrm{P}$ fertilizer on all days of sampling. While lower Fe-P content was recorded in the $\mathrm{P}_{0}$ level of $\mathrm{P}$ fertilizer at all days after incubation (Fig. 4(b)). The individual effect of sources of phosphorus was found non-significant $(P<0.05)$ on $\mathrm{Fe}-\mathrm{P}$ at all incubation intervals (Fig. 4(c)), but
MAP registered higher value of Fe-P (65.36 $\mathrm{mg} / \mathrm{kg}$ ) content in the soil as compared to DAP. Addition of $10 \mathrm{t} /$ ha FYM the magnitude of increased in Fe-P content $(67.26 \mathrm{mg} / \mathrm{kg})$ was significantly $(P<0.05)$ increased over control at $1^{\text {st }}, 3^{\text {rd }}, 5^{\text {th }}, 7^{\text {th }}$ and $14^{\text {th }}$ DAI (Fig. $4(d))$.

\section{Calcium-P (mg/kg)}

Figure 5 showed that maximum concentration of Ca-P content was recorded in $\mathrm{W}_{1}(192.62$ $\mathrm{mg} / \mathrm{kg}$ ) level of moisture regime whereas, minimum concentration in $\mathrm{W}_{3} \quad(180.29$ $\mathrm{mg} / \mathrm{kg}$ ) level of moisture regime (Fig. 5(a)). the maximum concentration of Ca-P (192.96 $\mathrm{mg} / \mathrm{kg}$ ) content was recorded with an application of $2.68 \mathrm{mg} \quad \mathrm{P} / 100 \mathrm{~g}$ soil phosphorus while lower concentration Ca-P content $(176.86 \mathrm{mg} / \mathrm{kg})$ recorded in $0.00 \mathrm{mg}$ P/kg 100 g soil (Fig. 5(b)). Likewise, the application of organic manure significantly $(P<0.05)$ increased the status of Ca-P content in the soil, the application of FYM (10 t/ha) significantly $(P<0.05)$ increased the build-up of Ca-P content $(187.86 \mathrm{mg} / \mathrm{kg})$ as compared to without FYM (Figure 5(d)).

\section{Occluded -P (mg/kg)}

The data presented in figure 6 shows that the Occluded-P fraction ranged from 9.29 to $12.97 \mathrm{mg} / \mathrm{kg}$ in the treatment moisture regime. However, the application of $100 \%$ available water capacity showed higher Occluded-P $(12.97 \mathrm{mg} / \mathrm{kg})$ content compared to $\mathrm{W}_{2}$ and $\mathrm{W}_{3}$ (Fig. 6(a)). Occluded-P measured at $1^{\text {st }}, 3^{\text {rd }}, 5^{\text {th }}, 7^{\text {th }}$ and $14^{\text {th }} \mathrm{DAI}$, respectively as influenced by different levels of phosphorus was significant $(P<0.05)$. Among the different levels of phosphorus, the application of $2.68 \mathrm{mg} \mathrm{P} / 100 \mathrm{~g}$ soil recorded the highest Occluded-P $(12.30 \mathrm{mg} / \mathrm{kg})$ at mean of $1^{\text {st }}, 3^{\text {rd }}, 5^{\text {th }}, 7^{\text {th }}$ and $14^{\text {th }}$ DAI, respectively as compared to $\mathrm{P}_{1}$ and $\mathrm{P}_{0}$ (Fig. (b)). FYM had a significant $(P<0.05)$ 
influence on Occluded-P recorded at all incubation intervals. The highest Occluded-P $(11.24 \mathrm{mg} / \mathrm{kg})$ content was observed with treatment $\mathrm{M}_{1}(10 \mathrm{t} / \mathrm{ha})$ at all days after sampling as compared to without application of 0 t/ha (Fig. 6(d)). Occluded-P values were found to increase during the initial period of incubation, but later these values were found to decrease in all the treatments.

\section{Reductant-P (mg/kg)}

A critical examination of data depicted in Figure 7 revealed that moisture regimes produced a significant $(P<0.05)$ effect in Reductant-P content at $1^{\text {st }}, 3^{\text {rd }}, 5^{\text {th }}, 7^{\text {th }}$ and $14^{\text {th }}$ DAI. Application of $100 \%$ available water capacity $\left(\mathrm{W}_{1}\right)$ in different incubation intervals recorded the maximum Reductant-P (42.68 $\mathrm{mg} / \mathrm{kg}$ ) content as compared to other moisture regimes at different days after sampling, the lowest concentration of Reductant-P was recorded with $25 \%$ available water capacity (Fig. 7 (a)). A significant $(P<0.05)$ increase in Reductant-P content at $1^{\text {st }}, 3^{\text {rd }}, 5^{\text {th }}, 7^{\text {th }}$, and $14^{\text {th }}$ DAI was observed due to an increase in levels of phosphorus. Among the different levels of phosphorus, the application of 2.68 $\mathrm{mg} \mathrm{P} / 100 \mathrm{~g}$ soil recorded the highest concentration of the Reductant-P (40.95 $\mathrm{mg} / \mathrm{kg}$ ) rate and proved its superiority to the rest of the treatments during all days after sampling (Fig. 7 (b)). Addition of FYM @ 10 t/ha recorded maximum concentration of Reductant-P $(39.76 \mathrm{mg} / \mathrm{kg})$ during $1^{\text {st }}, 3^{\text {rd }}, 5^{\text {th }}$, $7^{\text {th }}$, and $14^{\text {th }}$ DAI, respectively, which was significantly $(P<0.05)$ superior over no addition of FYM (Fig. 7(d)).

\section{Organic-P (mg/kg)}

The Organic-P content in soil was significantly $(P<0.05)$ influenced by moisture regimes, levels of $\mathrm{P}$ carriers and FYM was recorded at $1^{\text {st }}, 3^{\text {rd }}, 5^{\text {th }}, 7^{\text {th }}$ and $14^{\text {th }}$ DAI which was graphically depicted in Figure 8. The overall content of Organic-P was increased up to $7^{\text {th }}$ DAI, then decreasing Organic-P content up to $14^{\text {th }}$ DAI. In the case of Organic-P, treatment $\mathrm{W}_{1}$ recorded significantly $(P<0.05)$ highest concentration of Organic-P (102.41, $114.63,129.78,129.47$ and $120.52 \mathrm{mg} / \mathrm{kg}$ ) during $1^{\text {st }}$ to $14^{\text {th }}$ DAI, respectively (Fig. 8), the lowest Organic-P content $(76.29,81.22$, $89.60,92.51$ and $90.31 \mathrm{mg} / \mathrm{kg}$ ) was observed under the application of $25 \%$ available water capacity treatment during the $1^{\text {st }}, 3^{\text {rd }}, 5^{\text {th }}, 7^{\text {th }}$ and $14^{\text {th }}$ DAI, respectively. Phosphatic fertilizer treatments had a significant $(P<0.05)$ influence on Organic-P content in soil during all days after sampling. Significantly $(P<0.05)$ the highest Organic-P content to the tune of 91.49, 98.99, 109.53, 110.83 and 104.65 $\mathrm{mg} / \mathrm{kg}$ was noted under treatment $2.68 \mathrm{mg}$ P/100 g soil during $1^{\text {st }}, 3^{\text {rd }}, 5^{\text {th }}, 7^{\text {th }}$ and $14^{\text {th }}$ DAI, respectively (Fig. 8), significantly $(P<0.05)$ the lowest Organic-P content $(83.57$, $91.07,101.61,102.91$ and $96.73 \mathrm{mg} / \mathrm{kg}$ ) was observed with treatment $\mathrm{P}_{0}$. Sources of phosphorus did not cause a significant $(P<0.05)$ effect on Organic-P content in soil during all days after sampling (Fig. 8), the numerically higher concentration of Organic$P$ content was observed with the addition of MAP $(99.60 \mathrm{mg} / \mathrm{kg})$, while the addition of DAP gave the lower value $(98.84 \mathrm{mg} / \mathrm{kg})$. Addition of $10 \mathrm{t} /$ ha FYM, the magnitude of increase in mean Organic-P content (100.86 $\mathrm{mg} / \mathrm{kg}$ ) was observed as compared to control at $1^{\text {st }}, 3^{\text {rd }}, 5^{\text {th }}, 7^{\text {th }}$ and $14^{\text {th }}$ DAI (Fig. 8).

\section{Inorganic-P (mg/kg)}

It is apparent from the data of Figure 9 that there was a significant $(P<0.05)$ difference due to moisture regimes concerning Inorganic-P. A perusal of data indicated that the application of $100 \%$ available water capacity $\left(\mathrm{W}_{1}\right)$ produced highest Inorganic-P that was $378.19 \mathrm{mg} / \mathrm{kg}$ during all days after sampling, respectively. While, minimum Inorganic-P content was recorded with $25 \%$ 
available water capacity $\left(\mathrm{W}_{3}\right)$ at $1^{\text {st }}, 3^{\text {rd }}, 5^{\text {th }}, 7^{\text {th }}$ and $14^{\text {th }}$ DAI. Inorganic-P due to application of $\mathrm{W}_{1}$ moisture regime was increased 12.02 percent, respectively over the $\mathrm{W}_{3}$ moisture regime on a mean data basis (Fig. 9(a)). Application of $2.68 \mathrm{mg} \quad \mathrm{P} / 100 \mathrm{~g}$ soil significantly $(P<0.05)$ higher Inorganic-P over the $1.34 \mathrm{mg} \mathrm{P} / 100 \mathrm{~g}$ soil and $0.00 \mathrm{mg}$
$\mathrm{P} / 100 \mathrm{~g}$ soil during individual days after sampling. Significantly $(P<0.05)$ highest concentration of Inorganic-P $(378.70 \mathrm{mg} / \mathrm{kg})$ was obtained with an application of $2.68 \mathrm{mg}$ $\mathrm{P} / 100 \mathrm{~g}$ soil at $1^{\text {st }}, 3^{\text {rd }}, 5^{\text {th }}, 7^{\text {th }}$ and $14^{\text {th }}$ DAI, respectively. The behavior of Inorganic-P content in soil was in the order of $\mathrm{P}_{0}<\mathrm{P}_{1}<\mathrm{P}_{2}$ (Fig. 9(b)).

Table.1 Details of treatment combinations

\begin{tabular}{|c|c|c|c|c|c|c|c|c|c|}
\hline $\mathbf{T}_{1}$ & $\mathbf{M}_{0} \mathbf{W}_{1} \mathbf{S}_{1} \mathbf{P}_{0} \mathbf{I}_{0}$ & $\mathbf{T}_{37}$ & $\mathbf{M}_{0} \mathrm{~W}_{1} \mathbf{S}_{1} \mathbf{P}_{0} \mathbf{I}_{1}$ & $\mathbf{T}_{73}$ & $\mathbf{M}_{0} \mathbf{W}_{1} \mathbf{S}_{1} \mathbf{P}_{0} \mathbf{I}_{2}$ & $T_{109}$ & $\mathbf{M}_{0} \mathbf{W}_{1} \mathbf{S}_{1} \mathbf{P}_{0} \mathbf{I}_{3}$ & $\mathbf{T}_{145}$ & $\mathbf{M}_{0} \mathbf{W}_{1} \mathbf{S}_{1} \mathbf{P}_{0} \mathbf{I}_{4}$ \\
\hline $\mathbf{T}_{2}$ & $\mathrm{M}_{0} \mathrm{~W}_{2} \mathrm{~S}_{1} \mathrm{P}_{0} \mathrm{I}_{0}$ & $\mathrm{~T}_{38}$ & $\mathrm{M}_{0} \mathrm{~W}_{2} \mathrm{~S}_{1} \mathrm{P}_{0} \mathrm{I}_{1}$ & $\mathrm{~T}_{74}$ & $\mathrm{M}_{0} \mathrm{~W}_{2} \mathrm{~S}_{1} \mathrm{P}_{0} \mathrm{I}_{2}$ & $\mathrm{~T}_{110}$ & $\mathrm{M}_{0} \mathrm{~W}_{2} \mathrm{~S}_{1} \mathrm{P}_{0} \mathrm{I}_{3}$ & $\mathrm{~T}_{146}$ & $\mathrm{M}_{0} \mathrm{~W}_{2} \mathrm{~S}_{1} \mathrm{P}_{0} \mathrm{I}_{4}$ \\
\hline $\mathbf{T}_{3}$ & $\mathrm{M}_{0} \mathrm{~W}_{3} \mathrm{~S}_{1} \mathrm{P}_{0} \mathrm{I}_{0}$ & $\mathrm{~T}_{39}$ & $\mathrm{M}_{0} \mathrm{~W}_{3} \mathrm{~S}_{1} \mathrm{P}_{0} \mathrm{I}_{1}$ & $\mathrm{~T}_{75}$ & $\mathrm{M}_{0} \mathrm{~W}_{3} \mathrm{~S}_{1} \mathrm{P}_{0} \mathrm{I}_{2}$ & $\mathrm{~T}_{111}$ & $\mathrm{M}_{0} \mathrm{~W}_{3} \mathrm{~S}_{1} \mathrm{P}_{0} \mathrm{I}_{3}$ & $\mathrm{~T}_{147}$ & $\mathrm{M}_{0} \mathrm{~W}_{3} \mathrm{~S}_{1} \mathrm{P}_{0} \mathrm{I}_{4}$ \\
\hline $\mathbf{T}_{4}$ & $\mathrm{M}_{0} \mathrm{~W}_{1} \mathrm{~S}_{2} \mathrm{P}_{0} \mathrm{I}_{0}$ & $\mathrm{~T}_{40}$ & $\mathrm{M}_{0} \mathrm{~W}_{1} \mathrm{~S}_{2} \mathrm{P}_{0} \mathrm{I}_{1}$ & $\mathrm{~T}_{76}$ & $\mathrm{M}_{0} \mathrm{~W}_{1} \mathrm{~S}_{2} \mathrm{P}_{0} \mathrm{I}_{2}$ & $\mathrm{~T}_{112}$ & $\mathrm{M}_{0} \mathrm{~W}_{1} \mathrm{~S}_{2} \mathrm{P}_{0} \mathrm{I}_{3}$ & $\mathrm{~T}_{148}$ & $\mathrm{M}_{0} \mathrm{~W}_{1} \mathrm{~S}_{2} \mathrm{P}_{0} \mathrm{I}_{4}$ \\
\hline $\mathbf{T}_{5}$ & $\mathrm{M}_{0} \mathrm{~W}_{2} \mathrm{~S}_{2} \mathrm{P}_{0} \mathrm{I}_{0}$ & $\mathrm{~T}_{41}$ & $\mathrm{M}_{0} \mathrm{~W}_{2} \mathrm{~S}_{2} \mathrm{P}_{0} \mathrm{I}_{1}$ & $\mathrm{~T}_{77}$ & $\mathrm{M}_{0} \mathrm{~W}_{2} \mathrm{~S}_{2} \mathrm{P}_{0} \mathrm{I}_{2}$ & $\mathrm{~T}_{113}$ & $\mathrm{M}_{0} \mathrm{~W}_{2} \mathrm{~S}_{2} \mathrm{P}_{0} \mathrm{I}_{3}$ & $\mathrm{~T}_{149}$ & $\mathrm{M}_{0} \mathrm{~W}_{2} \mathrm{~S}_{2} \mathrm{P}_{0} \mathrm{I}_{4}$ \\
\hline $\mathbf{T}_{6}$ & $\mathrm{M}_{0} \mathrm{~W}_{3} \mathrm{~S}_{2} \mathrm{P}_{0} \mathrm{I}_{0}$ & $\mathrm{~T}_{42}$ & $\mathrm{M}_{0} \mathrm{~W}_{3} \mathrm{~S}_{2} \mathrm{P}_{0} \mathrm{I}_{1}$ & $\mathrm{~T}_{78}$ & $\mathrm{M}_{0} \mathrm{~W}_{3} \mathrm{~S}_{2} \mathrm{P}_{0} \mathrm{I}_{2}$ & $\mathrm{~T}_{114}$ & $\mathrm{M}_{0} \mathrm{~W}_{3} \mathrm{~S}_{2} \mathrm{P}_{0} \mathrm{I}_{3}$ & $\mathrm{~T}_{150}$ & $\mathrm{M}_{0} \mathrm{~W}_{3} \mathrm{~S}_{2} \mathrm{P}_{0} \mathrm{I}_{4}$ \\
\hline $\mathbf{T}_{7}$ & $\mathrm{M}_{0} \mathrm{~W}_{1} \mathrm{~S}_{1} \mathrm{P}_{1} \mathrm{I}_{0}$ & $\mathrm{~T}_{43}$ & $\mathrm{M}_{0} \mathrm{~W}_{1} \mathrm{~S}_{1} \mathrm{P}_{1} \mathrm{I}_{1}$ & $\mathrm{~T}_{79}$ & $\mathrm{M}_{0} \mathrm{~W}_{1} \mathrm{~S}_{1} \mathrm{P}_{1} \mathrm{I}_{2}$ & $\mathrm{~T}_{115}$ & $\mathrm{M}_{0} \mathrm{~W}_{1} \mathrm{~S}_{1} \mathrm{P}_{1} \mathrm{I}_{3}$ & $\mathrm{~T}_{151}$ & $\mathrm{M}_{0} \mathrm{~W}_{1} \mathrm{~S}_{1} \mathrm{P}_{1} \mathrm{I}_{4}$ \\
\hline $\mathbf{T}_{8}$ & $\mathrm{M}_{0} \mathrm{~W}_{2} \mathrm{~S}_{1} \mathrm{P}_{1} \mathrm{I}_{0}$ & $\mathrm{~T}_{44}$ & $\mathrm{M}_{0} \mathrm{~W}_{2} \mathrm{~S}_{1} \mathrm{P}_{1} \mathrm{I}_{1}$ & $\mathrm{~T}_{80}$ & $\mathrm{M}_{0} \mathrm{~W}_{2} \mathrm{~S}_{1} \mathrm{P}_{1} \mathrm{I}_{2}$ & $\mathrm{~T}_{116}$ & $\mathrm{M}_{0} \mathrm{~W}_{2} \mathrm{~S}_{1} \mathrm{P}_{1} \mathrm{I}_{3}$ & $\mathrm{~T}_{152}$ & $\mathrm{M}_{0} \mathrm{~W}_{2} \mathrm{~S}_{1} \mathrm{P}_{1} \mathrm{I}_{4}$ \\
\hline $\mathbf{T}_{9}$ & $\mathrm{M}_{0} \mathrm{~W}_{3} \mathrm{~S}_{1} \mathrm{P}_{1} \mathrm{I}_{0}$ & $\mathrm{~T}_{45}$ & $\mathrm{M}_{0} \mathrm{~W}_{3} \mathrm{~S}_{1} \mathrm{P}_{1} \mathrm{I}_{1}$ & $\mathrm{~T}_{81}$ & $\mathrm{M}_{0} \mathrm{~W}_{3} \mathrm{~S}_{1} \mathrm{P}_{1} \mathrm{I}_{2}$ & $\mathrm{~T}_{117}$ & $\mathrm{M}_{0} \mathrm{~W}_{3} \mathrm{~S}_{1} \mathrm{P}_{1} \mathrm{I}_{3}$ & $\mathrm{~T}_{153}$ & $\mathrm{M}_{0} \mathrm{~W}_{3} \mathrm{~S}_{1} \mathrm{P}_{1} \mathrm{I}_{4}$ \\
\hline$T_{10}$ & $\mathrm{M}_{0} \mathrm{~W}_{1} \mathrm{~S}_{2} \mathrm{P}_{1} \mathrm{I}_{0}$ & $\mathrm{~T}_{46}$ & $\mathrm{M}_{0} \mathrm{~W}_{1} \mathrm{~S}_{2} \mathrm{P}_{1} \mathrm{I}_{1}$ & $\mathrm{~T}_{82}$ & & $\mathrm{~T}_{118}$ & & $\mathrm{~T}_{154}$ & $\mathrm{M}_{0} \mathrm{~W}_{1} \mathrm{~S}_{2} \mathrm{P}_{1} \mathrm{I}_{4}$ \\
\hline $\mathbf{T}_{11}$ & $\mathrm{M}_{0} \mathrm{~W}_{2} \mathrm{~S}_{2} \mathrm{P}_{1} \mathrm{I}_{0}$ & $\mathrm{~T}_{47}$ & $\mathrm{M}_{0} \mathrm{~W}_{2} \mathrm{~S}_{2} \mathrm{P}_{1} \mathrm{I}_{1}$ & $\mathrm{~T}_{83}$ & $\mathrm{M}_{0} \mathrm{~W}_{2} \mathrm{~S}_{2} \mathrm{P}_{1} \mathrm{I}_{2}$ & $\mathrm{~T}_{119}$ & $\mathrm{M}_{0} \mathrm{~W}_{2} \mathrm{~S}_{2} \mathrm{P}_{1} \mathrm{I}_{3}$ & $\mathrm{~T}_{155}$ & $\mathrm{M}_{0} \mathrm{~W}_{2} \mathrm{~S}_{2} \mathrm{P}_{1} \mathrm{I}_{4}$ \\
\hline $\mathbf{T}_{12}$ & $\mathrm{M}_{0} \mathrm{~W}_{3} \mathrm{~S}_{2} \mathrm{P}_{1} \mathrm{I}_{0}$ & $\mathrm{~T}_{48}$ & $\mathrm{M}_{0} \mathrm{~W}_{3} \mathrm{~S}_{2} \mathrm{P}_{1} \mathrm{I}_{1}$ & $\mathrm{~T}_{84}$ & $\mathrm{M}_{0} \mathrm{~W}_{3} \mathrm{~S}_{2} \mathrm{P}_{1} \mathrm{I}_{2}$ & $\mathrm{~T}_{120}$ & $\mathrm{M}_{0} \mathrm{~W}_{3} \mathrm{~S}_{2} \mathrm{P}_{1} \mathrm{I}_{3}$ & $\mathrm{~T}_{156}$ & $\mathrm{M}_{0} \mathrm{~W}_{3} \mathrm{~S}_{2} \mathrm{P}_{1} \mathrm{I}_{4}$ \\
\hline $\mathbf{T}_{13}$ & $\mathrm{M}_{0} \mathrm{~W}_{1} \mathrm{~S}_{1} \mathrm{P}_{2} \mathrm{I}_{0}$ & $\mathrm{~T}_{49}$ & $\mathrm{M}_{0} \mathrm{~W}_{1} \mathrm{~S}_{1} \mathrm{P}_{2} \mathrm{I}_{1}$ & $\mathrm{~T}_{85}$ & $\mathrm{M}_{0} \mathrm{~W}_{1} \mathrm{~S}_{1} \mathrm{P}_{2} \mathrm{I}_{2}$ & $\mathrm{~T}_{121}$ & $\mathrm{M}_{0} \mathrm{~W}_{1} \mathrm{~S}_{1} \mathrm{P}_{2} \mathrm{I}_{3}$ & $\mathrm{~T}_{157}$ & $\mathrm{M}_{0} \mathrm{~W}_{1} \mathrm{~S}_{1} \mathrm{P}_{2} \mathrm{I}_{4}$ \\
\hline$T_{14}$ & $\mathrm{M}_{0} \mathrm{~W}_{2} \mathrm{~S}_{1} \mathrm{P}_{2} \mathrm{I}_{0}$ & $\mathrm{~T}_{50}$ & $\mathrm{M}_{0} \mathrm{~W}_{2} \mathrm{~S}_{1} \mathrm{P}_{2} \mathrm{I}_{1}$ & $\mathrm{~T}_{86}$ & $\mathrm{M}_{0} \mathrm{~W}_{2} \mathrm{~S}_{1} \mathrm{P}_{2} \mathrm{I}_{2}$ & & $\mathrm{M}_{0} \mathrm{~W}_{2} \mathrm{~S}_{1} \mathrm{P}_{2} \mathrm{I}_{3}$ & $\mathrm{~T}_{158}$ & $\mathrm{M}_{0} \mathrm{~W}_{2} \mathrm{~S}_{1} \mathrm{P}_{2} \mathrm{I}_{4}$ \\
\hline$T_{15}$ & $\mathrm{M}_{0} \mathrm{~W}_{3} \mathrm{~S}_{1} \mathrm{P}_{2} \mathrm{I}_{0}$ & $\mathrm{~T}_{51}$ & & $\mathrm{~T}_{87}$ & & & & $\mathrm{~T}_{159}$ & \\
\hline$T_{16}$ & $\mathrm{M}_{0} \mathrm{~W}_{1} \mathrm{~S}_{2} \mathrm{P}_{2} \mathrm{I}_{0}$ & $\mathrm{~T}_{52}$ & $\mathrm{M}_{0} \mathrm{~W}_{1} \mathrm{~S}_{2} \mathrm{P}_{2} \mathrm{I}_{1}$ & $\mathrm{~T}_{88}$ & $\mathrm{M}_{0} \mathrm{~W}_{1} \mathrm{~S}_{2} \mathrm{P}_{2} \mathrm{I}_{2}$ & $\mathrm{~T}_{124}$ & & $\mathrm{~T}_{160}$ & \\
\hline $\mathbf{T}_{17}$ & $\mathrm{M}_{0} \mathrm{~W}_{2} \mathrm{~S}_{2} \mathrm{P}_{2} \mathrm{I}_{0}$ & $\mathrm{~T}_{53}$ & $\mathrm{M}_{0} \mathrm{~W}_{2} \mathrm{~S}_{2} \mathrm{P}_{2} \mathrm{I}_{1}$ & $\mathrm{~T}_{89}$ & $\mathrm{M}_{0} \mathrm{~W}_{2} \mathrm{~S}_{2} \mathrm{P}_{2} \mathrm{I}_{2}$ & $\mathrm{~T}_{125}$ & $\mathrm{M}_{0} \mathrm{~W}_{2} \mathrm{~S}_{2} \mathrm{P}_{2} \mathrm{I}_{3}$ & $\mathrm{~T}_{161}$ & $\mathrm{M}_{0} \mathrm{~W}_{2} \mathrm{~S}_{2} \mathrm{P}_{2} \mathrm{I}_{4}$ \\
\hline $\mathbf{T}_{18}$ & $\mathrm{M}_{0} \mathrm{~W}_{3} \mathrm{~S}_{2} \mathrm{P}_{2} \mathrm{I}_{0}$ & $\mathrm{~T}_{54}$ & $\mathrm{M}_{0} \mathrm{~W}_{3} \mathrm{~S}$ & $\mathrm{~T}_{90}$ & $\mathrm{M}_{0} \mathrm{~W}_{3} \mathrm{~S}$ & $\mathrm{~T}_{126}$ & $\mathrm{M}_{0} \mathrm{~W}_{3} \mathrm{~S}_{2} \mathrm{P}_{2} \mathrm{I}_{3}$ & $\mathrm{~T}_{162}$ & ${ }_{3} \mathrm{~S}_{2} \mathrm{P}_{2} \mathrm{I}_{4}$ \\
\hline $\mathrm{T}_{19}$ & $\mathrm{M}_{1} \mathrm{~W}_{1} \mathrm{~S}_{1} \mathrm{P}_{0} \mathrm{I}_{0}$ & $\mathrm{~T}_{55}$ & $\mathrm{M}_{1} \mathrm{~W}_{1} \mathrm{~S}_{1} \mathrm{P}_{0} \mathrm{I}_{1}$ & $\mathrm{~T}_{91}$ & $\mathrm{M}_{1} \mathrm{~W}_{1} \mathrm{~S}_{1} \mathrm{P}_{0} \mathrm{I}_{2}$ & $\mathrm{~T}_{127}$ & $\mathrm{M}_{1} \mathrm{~W}_{1} \mathrm{~S}_{1} \mathrm{P}_{0} \mathrm{I}_{3}$ & $\mathrm{~T}_{163}$ & $\mathrm{M}_{1} \mathrm{~W}_{1} \mathrm{~S}_{1} \mathrm{P}_{0} \mathrm{I}_{4}$ \\
\hline $\mathbf{T}_{20}$ & $\mathrm{M}_{1} \mathrm{~W}_{2} \mathrm{~S}_{1} \mathrm{P}_{0} \mathrm{I}_{0}$ & $\mathrm{~T}_{56}$ & & $\mathrm{~T}_{92}$ & & $\mathrm{~T}_{128}$ & & $\mathrm{~T}_{164}$ & \\
\hline $\mathbf{T}_{21}$ & $\mathrm{M}_{1} \mathrm{~W}_{3} \mathrm{~S}_{1} \mathrm{P}_{0} \mathrm{I}_{0}$ & $\mathrm{~T}_{57}$ & $\mathrm{M}_{1} \mathrm{~W}_{3} \mathrm{~S}_{1} \mathrm{P}_{0} \mathrm{I}_{1}$ & $\mathrm{~T}_{93}$ & & $\mathrm{~T}_{129}$ & & $\mathrm{~T}_{165}$ & $\mathrm{M}_{1} \mathrm{~W}_{3} \mathrm{~S}_{1} \mathrm{P}_{0} \mathrm{I}_{4}$ \\
\hline $\mathbf{T}_{22}$ & $\mathrm{M}_{1} \mathrm{~W}_{1} \mathrm{~S}_{2} \mathrm{P}_{0} \mathrm{I}_{0}$ & $\mathrm{~T}_{58}$ & & $\mathrm{~T}_{94}$ & & & & $\mathrm{~T}_{166}$ & \\
\hline $\mathbf{T}_{23}$ & $\mathrm{M}_{1} \mathrm{~W}_{2} \mathrm{~S}_{2} \mathrm{P}_{0} \mathrm{I}_{0}$ & $\mathrm{~T}_{59}$ & $\mathrm{M}_{1} \mathrm{~W}_{2} \mathrm{~S}_{2} \mathrm{P}_{0} \mathrm{I}_{1}$ & $\mathrm{~T}_{95}$ & $\mathrm{M}_{1} \mathrm{~W}_{2} \mathrm{~S}_{2} \mathrm{P}_{0} \mathrm{I}_{2}$ & $\mathrm{~T}_{131}$ & $\mathrm{M}_{1} \mathrm{~W}_{2} \mathrm{~S}_{2} \mathrm{P}_{0} \mathrm{I}_{3}$ & $\mathrm{~T}_{167}$ & $\mathrm{M}_{1} \mathrm{~W}_{2} \mathrm{~S}_{2} \mathrm{P}_{0} \mathrm{I}_{4}$ \\
\hline $\mathbf{T}_{24}$ & $\mathrm{M}_{1} \mathrm{~W}_{3} \mathrm{~S}_{2} \mathrm{P}_{0} \mathrm{I}_{0}$ & $\mathrm{~T}_{60}$ & $\mathrm{M}_{1} \mathrm{~W}_{3} \mathrm{~S}_{2} \mathrm{P}_{0} \mathrm{I}_{1}$ & $\mathrm{~T}_{96}$ & $\mathrm{M}_{1} \mathrm{~W}_{3} \mathrm{~S}_{2} \mathrm{P}_{0} \mathrm{I}_{2}$ & $\mathrm{~T}_{132}$ & $\mathrm{M}_{1} \mathrm{~W}_{3} \mathrm{~S}_{2} \mathrm{P}_{0} \mathrm{I}_{3}$ & $\mathrm{~T}_{168}$ & $\mathrm{M}_{1} \mathrm{~W}_{3} \mathrm{~S}_{2} \mathrm{P}_{0} \mathrm{I}_{4}$ \\
\hline$T_{25}$ & $\mathrm{M}_{1} \mathrm{~W}_{1} \mathrm{~S}_{1} \mathrm{P}_{1} \mathrm{I}_{0}$ & $\mathrm{~T}_{61}$ & $\mathrm{M}_{1} \mathrm{~W}_{1} \mathrm{~S}_{1} \mathrm{P}_{1} \mathrm{I}_{1}$ & $\mathrm{~T}_{97}$ & $\mathrm{M}_{1} \mathrm{~W}_{1} \mathrm{~S}_{1} \mathrm{P}_{1} \mathrm{I}_{2}$ & $\mathrm{~T}_{133}$ & $\mathrm{M}_{1} \mathrm{~W}_{1} \mathrm{~S}_{1} \mathrm{P}_{1} \mathrm{I}_{3}$ & $\mathrm{~T}_{169}$ & $\mathrm{M}_{1} \mathrm{~W}_{1} \mathrm{~S}_{1} \mathrm{P}_{1} \mathrm{I}_{4}$ \\
\hline $\mathbf{T}_{26}$ & $\mathrm{M}_{1} \mathrm{~W}_{2} \mathrm{~S}_{1} \mathrm{P}_{1} \mathrm{I}_{0}$ & $\mathrm{~T}_{62}$ & $\mathrm{M}_{1} \mathrm{~W}_{2} \mathrm{~S}_{1} \mathrm{P}_{1} \mathrm{I}_{1}$ & $\mathrm{~T}_{98}$ & $\mathrm{M}_{1} \mathrm{~W}_{2} \mathrm{~S}_{1} \mathrm{P}_{1} \mathrm{I}_{2}$ & $\mathrm{~T}_{134}$ & $\mathrm{M}_{1} \mathrm{~W}_{2} \mathrm{~S}_{1} \mathrm{P}_{1} \mathrm{I}_{3}$ & $\mathrm{~T}_{170}$ & $\mathrm{M}_{1} \mathrm{~W}_{2} \mathrm{~S}_{1} \mathrm{P}_{1} \mathrm{I}_{4}$ \\
\hline $\mathbf{T}_{27}$ & $\mathrm{M}_{1} \mathrm{~W}_{3} \mathrm{~S}_{1} \mathrm{P}_{1} \mathrm{I}_{0}$ & $\mathrm{~T}_{63}$ & $\mathrm{M}_{1} \mathrm{~W}_{3} \mathrm{~S}_{1} \mathrm{P}_{1} \mathrm{I}_{1}$ & $\mathrm{~T}_{99}$ & & & & $\mathrm{~T}_{171}$ & $\mathrm{M}_{1} \mathrm{~W}_{3} \mathrm{~S}_{1} \mathrm{P}_{1} \mathrm{I}_{4}$ \\
\hline$T_{28}$ & $\mathrm{M}_{1} \mathrm{~W}_{1} \mathrm{~S}_{2} \mathrm{P}_{1} \mathrm{I}_{0}$ & $\mathrm{~T}_{64}$ & $\mathrm{M}_{1} \mathrm{~W}_{1} \mathrm{~S}_{2} \mathrm{P}_{1} \mathrm{I}_{1}$ & $\mathrm{~T}_{100}$ & $\mathrm{M}_{1} \mathrm{~W}_{1} \mathrm{~S}_{2} \mathrm{P}_{1} \mathrm{I}_{2}$ & $\mathrm{~T}_{136}$ & $\mathrm{M}_{1} \mathrm{~W}_{1} \mathrm{~S}_{2} \mathrm{P}_{1} \mathrm{I}_{3}$ & $\mathrm{~T}_{172}$ & $\mathrm{M}_{1} \mathrm{~W}_{1} \mathrm{~S}_{2} \mathrm{P}_{1} \mathrm{I}_{4}$ \\
\hline$T_{29}$ & $\mathrm{M}_{1} \mathrm{~W}_{2} \mathrm{~S}_{2} \mathrm{P}_{1} \mathrm{I}_{0}$ & $\mathrm{~T}_{65}$ & $\mathrm{M}_{1} \mathrm{~W}_{2} \mathrm{~S}_{2} \mathrm{P}_{1} \mathrm{I}_{1}$ & $\mathrm{~T}_{101}$ & $\mathrm{M}_{1} \mathrm{~W}_{2} \mathrm{~S}_{2} \mathrm{P}_{1} \mathrm{I}_{2}$ & & & $\mathrm{~T}_{173}$ & $\mathrm{M}_{1} \mathrm{~W}_{2} \mathrm{~S}_{2} \mathrm{P}_{1} \mathrm{I}_{4}$ \\
\hline $\mathbf{T}_{30}$ & $\mathrm{M}_{1} \mathrm{~W}_{3} \mathrm{~S}_{2} \mathrm{P}_{1} \mathrm{I}_{0}$ & $\mathrm{~T}_{66}$ & $\mathrm{M}_{1} \mathrm{~W}_{3} \mathrm{~S}_{2} \mathrm{P}_{1} \mathrm{I}_{1}$ & $\mathrm{~T}_{102}$ & $\mathrm{M}_{1} \mathrm{~W}_{3} \mathrm{~S}_{2} \mathrm{P}_{1} \mathrm{I}_{2}$ & $\mathrm{~T}_{138}$ & $\mathrm{M}_{1} \mathrm{~W}_{3} \mathrm{~S}_{2} \mathrm{P}_{1} \mathrm{I}_{3}$ & $\mathrm{~T}_{174}$ & $\mathrm{M}_{1} \mathrm{~W}_{3} \mathrm{~S}_{2} \mathrm{P}_{1} \mathrm{I}_{4}$ \\
\hline $\mathbf{T}_{31}$ & $\mathrm{M}_{1} \mathrm{~W}_{1} \mathrm{~S}_{1} \mathrm{P}_{2} \mathrm{I}_{0}$ & $\mathrm{~T}_{67}$ & $\mathrm{M}_{1} \mathrm{~W}_{1} \mathrm{~S}_{1} \mathrm{P}_{2} \mathrm{I}_{1}$ & $\mathrm{~T}_{103}$ & $\mathrm{M}_{1} \mathrm{~W}_{1} \mathrm{~S}_{1} \mathrm{P}_{2} \mathrm{I}_{2}$ & $\mathrm{~T}_{139}$ & & $\mathrm{~T}_{175}$ & $\mathrm{M}_{1} \mathrm{~W}_{1} \mathrm{~S}_{1} \mathrm{P}_{2} \mathrm{I}_{4}$ \\
\hline $\mathbf{T}_{32}$ & $\mathrm{M}_{1} \mathrm{~W}_{2} \mathrm{~S}_{1} \mathrm{P}_{2} \mathrm{I}_{0}$ & $\mathrm{~T}_{68}$ & $\mathrm{M}_{1} \mathrm{~W}_{2} \mathrm{~S}_{1} \mathrm{P}_{2} \mathrm{I}_{1}$ & $\mathrm{~T}_{104}$ & $\mathrm{M}_{1} \mathrm{~W}_{2} \mathrm{~S}_{1} \mathrm{P}_{2} \mathrm{I}_{2}$ & $\mathrm{~T}_{140}$ & $\mathrm{M}_{1} \mathrm{~W}_{2} \mathrm{~S}_{1} \mathrm{P}_{2} \mathrm{I}_{3}$ & $\mathrm{~T}_{176}$ & $\mathrm{M}_{1} \mathrm{~W}_{2} \mathrm{~S}_{1} \mathrm{P}_{2} \mathrm{I}_{4}$ \\
\hline $\mathbf{T}_{33}$ & $\mathrm{M}_{1} \mathrm{~W}_{3} \mathrm{~S}_{1} \mathrm{P}_{2} \mathrm{I}_{0}$ & $\mathrm{~T}_{69}$ & $\mathrm{M}_{1} \mathrm{~W}_{3} \mathrm{~S}_{1} \mathrm{P}_{2} \mathrm{I}_{1}$ & $\mathrm{~T}_{105}$ & $\mathrm{M}_{1} \mathrm{~W}_{3} \mathrm{~S}_{1} \mathrm{P}_{2} \mathrm{I}_{2}$ & $\mathrm{~T}_{141}$ & $\mathrm{M}_{1} \mathrm{~W}_{3} \mathrm{~S}_{1} \mathrm{P}_{2} \mathrm{I}_{3}$ & $\mathrm{~T}_{177}$ & $\mathrm{M}_{1} \mathrm{~W}_{3} \mathrm{~S}_{1} \mathrm{P}_{2} \mathrm{I}_{4}$ \\
\hline $\mathbf{T}_{34}$ & $\mathrm{M}_{1} \mathrm{~W}_{1} \mathrm{~S}_{2} \mathrm{P}_{2} \mathrm{I}_{0}$ & $\mathrm{~T}_{70}$ & $\mathrm{M}_{1} \mathrm{~W}_{1} \mathrm{~S}_{2} \mathrm{P}_{2} \mathrm{I}_{1}$ & $\mathrm{~T}_{106}$ & $\mathrm{M}_{1} \mathrm{~W}_{1} \mathrm{~S}_{2} \mathrm{P}_{2} \mathrm{I}_{2}$ & $\mathrm{~T}_{142}$ & $\mathrm{M}_{1} \mathrm{~W}_{1} \mathrm{~S}_{2} \mathrm{P}_{2} \mathrm{I}_{3}$ & $\mathrm{~T}_{178}$ & $\mathrm{M}_{1} \mathrm{~W}_{1} \mathrm{~S}_{2} \mathrm{P}_{2} \mathrm{I}_{4}$ \\
\hline $\mathbf{T}_{35}$ & $\mathrm{M}_{1} \mathrm{~W}_{2} \mathrm{~S}_{2} \mathrm{P}_{2} \mathrm{I}_{0}$ & $\mathrm{~T}_{71}$ & $\mathrm{M}_{1} \mathrm{~W}_{2} \mathrm{~S}_{2} \mathrm{P}_{2} \mathrm{I}_{1}$ & $\mathrm{~T}_{107}$ & $\mathrm{M}_{1} \mathrm{~W}_{2} \mathrm{~S}_{2} \mathrm{P}_{2} \mathrm{I}_{2}$ & $\mathrm{~T}_{143}$ & $\mathrm{M}_{1} \mathrm{~W}_{2} \mathrm{~S}_{2} \mathrm{P}_{2} \mathrm{I}_{3}$ & $\mathrm{~T}_{179}$ & $\mathrm{M}_{1} \mathrm{~W}_{2} \mathrm{~S}_{2} \mathrm{P}_{2} \mathrm{I}_{4}$ \\
\hline $\mathbf{T}_{36}$ & $\mathrm{M}_{1} \mathrm{~W}_{3} \mathrm{~S}_{2} \mathrm{P}_{2} \mathrm{I}_{0}$ & $\mathrm{~T}_{72}$ & $\mathrm{M}_{1} \mathrm{~W}_{3} \mathrm{~S}_{2} \mathrm{P}_{2} \mathrm{I}_{1}$ & $\mathrm{~T}_{108}$ & $\mathrm{M}_{1} \mathrm{~W}_{3} \mathrm{~S}_{2} \mathrm{P}_{2} \mathrm{I}_{2}$ & $\mathrm{~T}_{144}$ & $\mathrm{M}_{1} \mathrm{~W}_{3} \mathrm{~S}_{2} \mathrm{P}_{2} \mathrm{I}_{3}$ & $\mathrm{~T}_{180}$ & $\mathrm{M}_{1} \mathrm{~W}_{3} \mathrm{~S}_{2} \mathrm{P}_{2} \mathrm{I}_{4}$ \\
\hline
\end{tabular}


Fig.1 Effect of moisture regimes (a), FYM (d) and levels of P (b) carriers (c) on available $\mathrm{P}_{2} \mathrm{O}_{5}$ in soil at different intervals of incubation

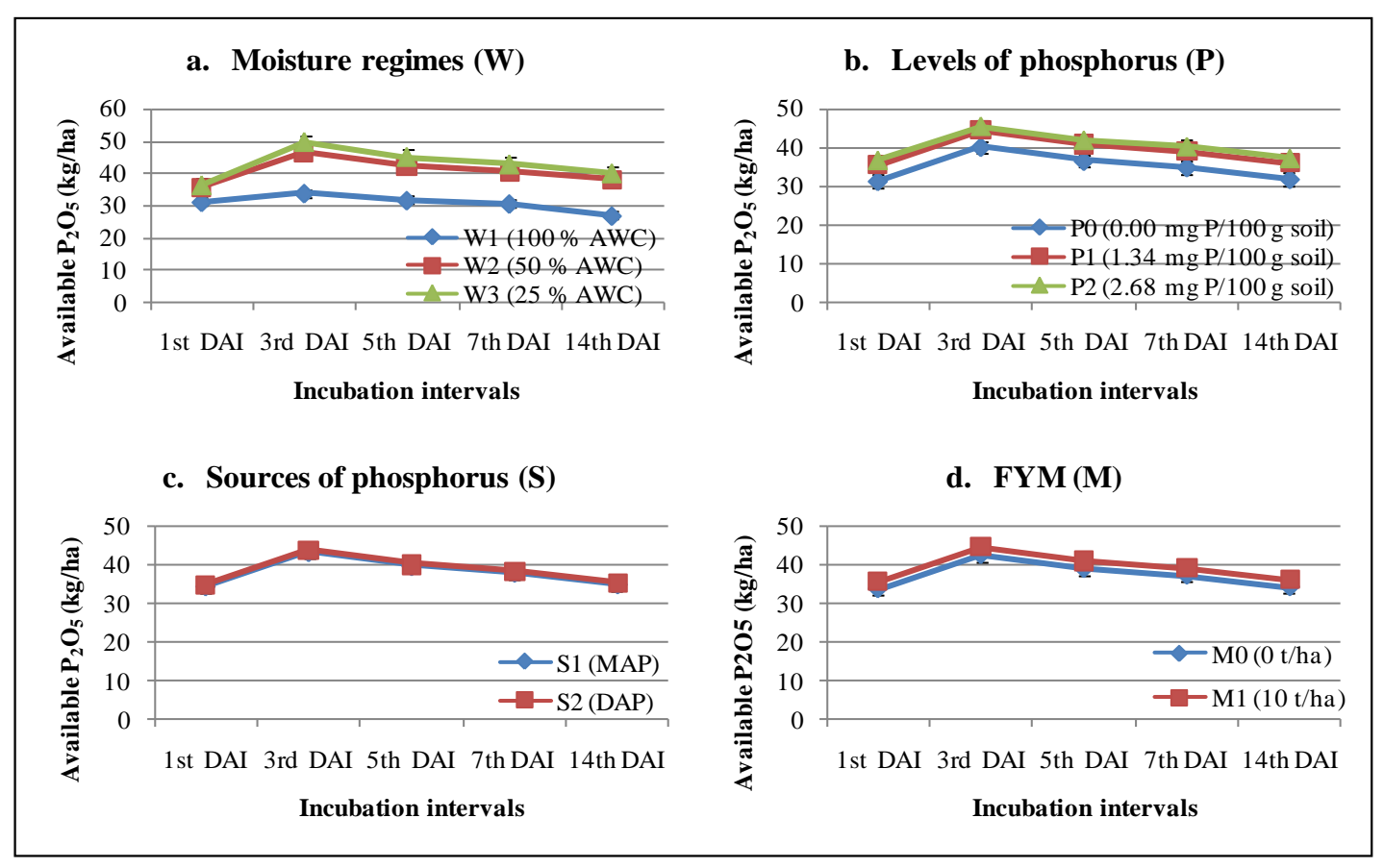

Fig.2 Effect of moisture regimes (a), FYM (d) and levels of P (b) carriers (c) on saloid-P in soil at different intervals of incubation

a. Moisture regimes (W)

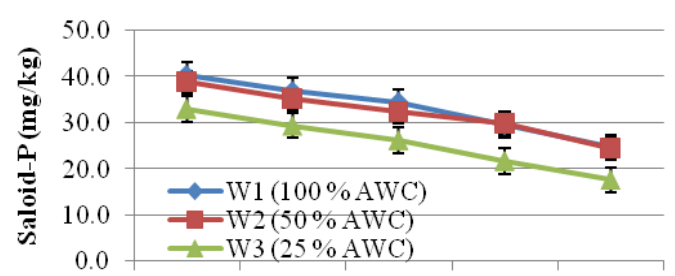

1st DAI 3rd DAI 5th DAI 7th DAI 14th DAI

Incubation intervals

c. Sources of phosphorus (S)

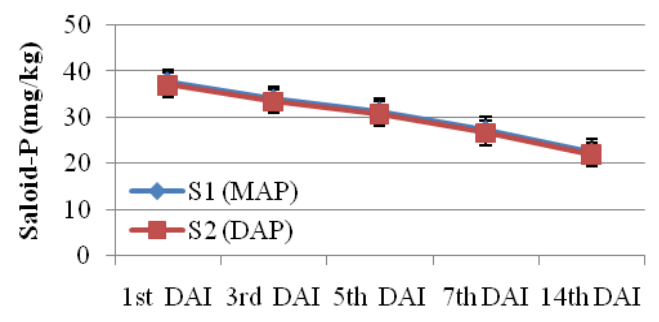

Incubation intervals b. Levels of phosphorus (P)

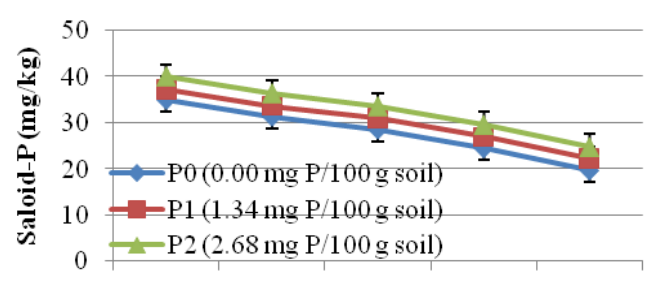

1st DAI 3rd DAI 5th DAI 7thDAI 14th DAI

Incubation intervals

d. FYM (M)

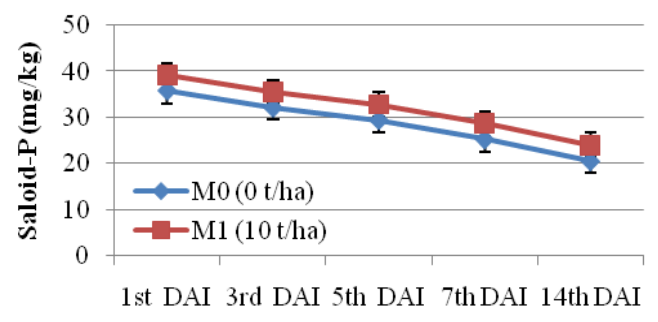

Incubation intervals 
Fig. 3 Effect of moisture regimes (a), FYM (d) and levels of P (b) carriers (c) on Al-P in soil at different intervals of incubation

a. Moisture regimes (W)

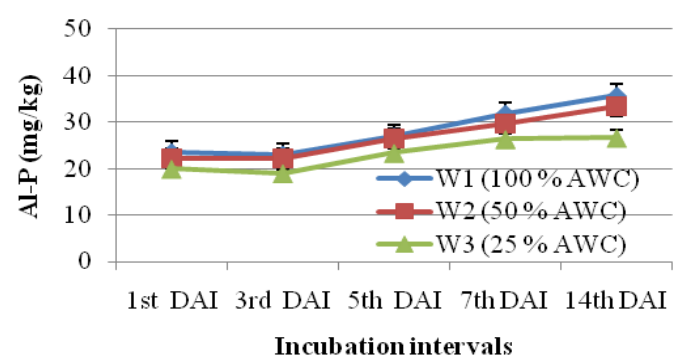

c. Sources of phosphorus (S)

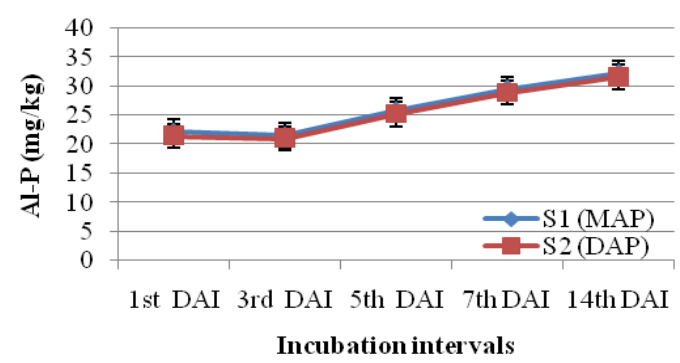

b. Levels of phosphorus (P)

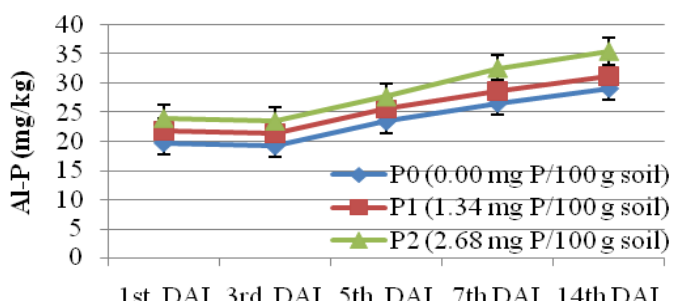

Incubation intervals

d. FYM (M)

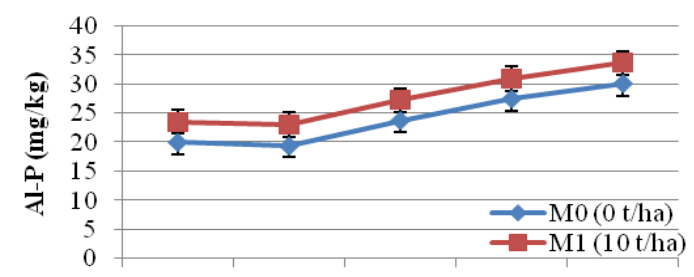

1st DAI 3rd DAI 5th DAI 7th DAI 14th DAI

Incubation intervals

Fig.4 Effect of moisture regimes (a), FYM (d) and levels of P (b) carriers (c) on Fe-P in soil at different intervals of incubation

a. Moisture regimes (W)

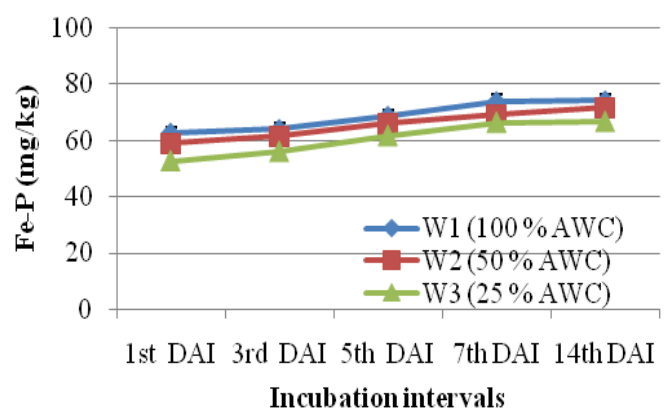

c. Sources of phosphorus (S)

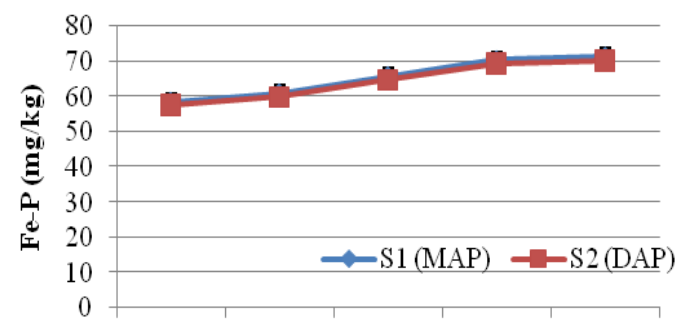

1st DAI 3rd DAI 5th DAI 7th DAI 14th DAI Incubation intervals b. Levels of phosphorus (P)

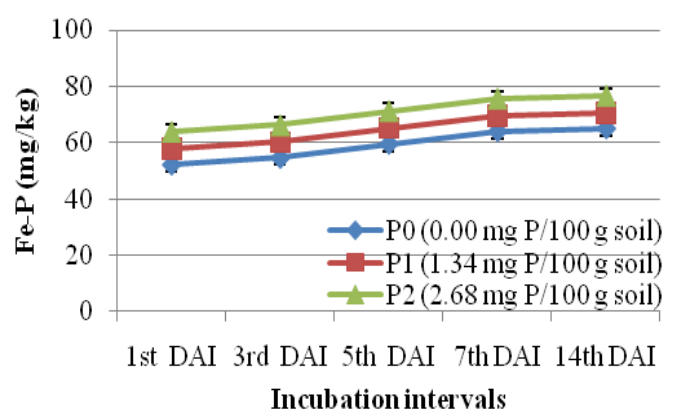

d. FYM (M)

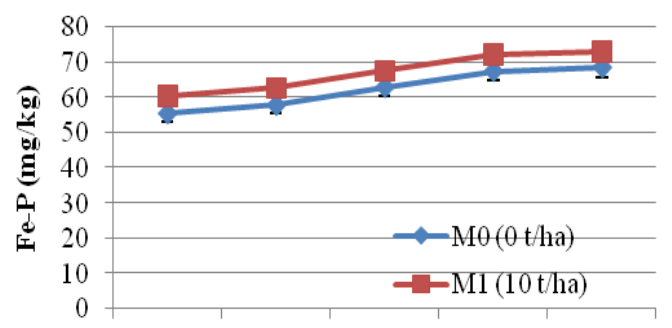

1st DAI 3rd DAI 5th DAI 7thDAI 14thDAI Incubation intervals 
Fig.5 Effect of moisture regimes (a), FYM (d) and levels of P (b) carriers (c) on Ca-P in soil at different intervals of incubation

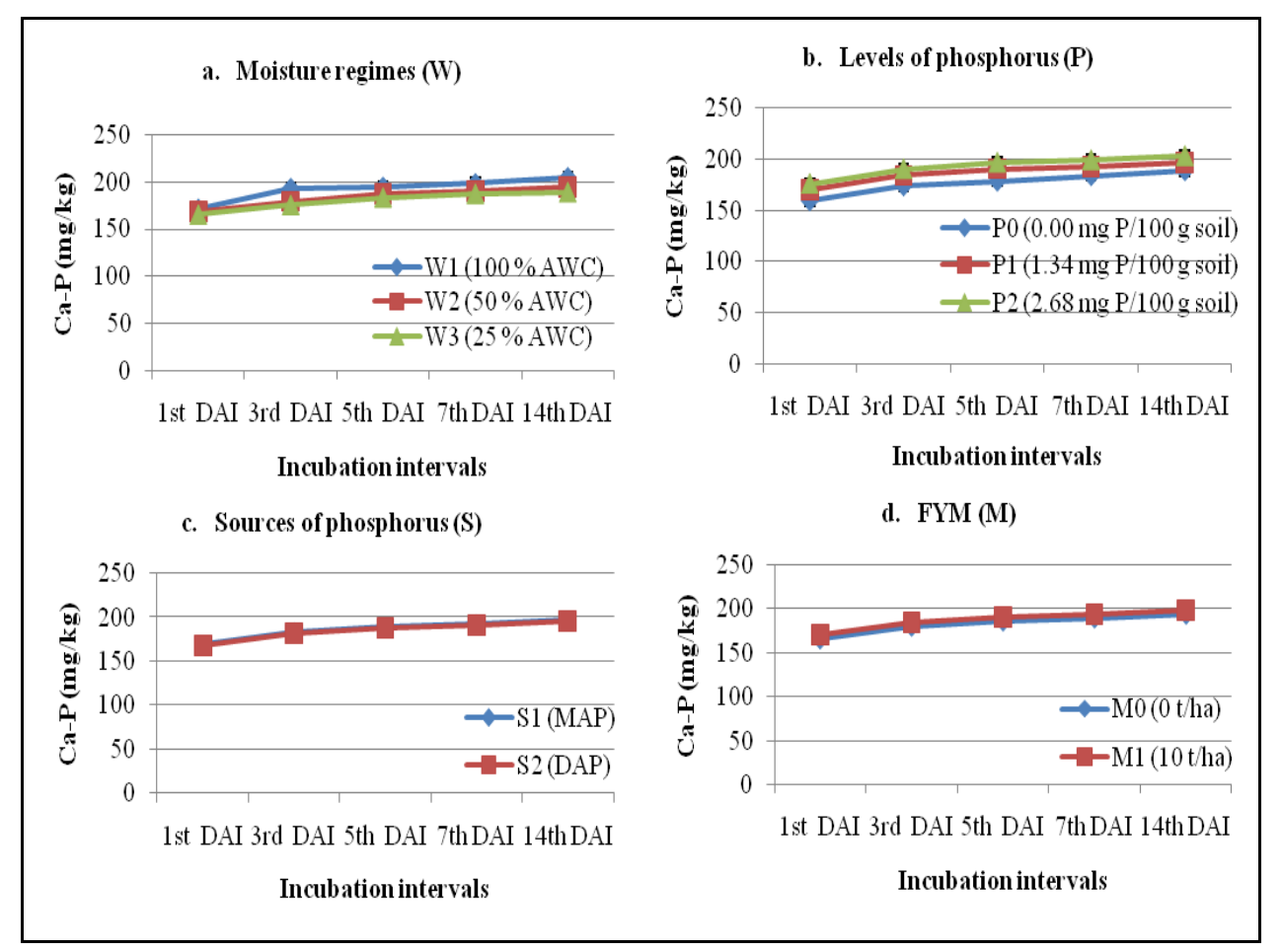

Fig.6 Effect of moisture regimes (a), FYM (d) and levels of P (b) carriers (c) on occluded-P in soil at different intervals of incubation

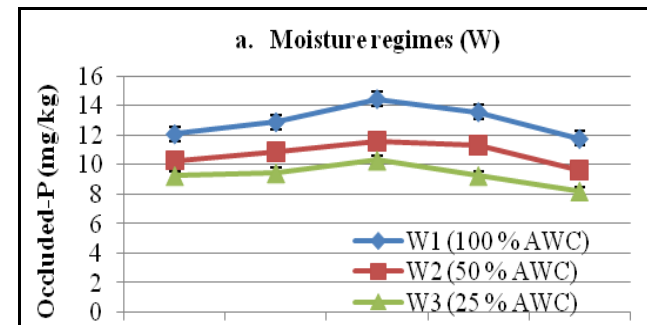

1st DAI 3rd DAI 5th DAI 7thDAI 14thDAI

Incubation intervals

c. Sources of phosphorus (S)

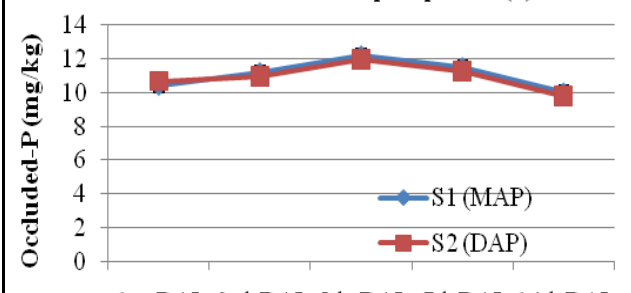

1st DAI 3rd DAI 5th DAI 7thDAI 14thDAI

Incubation intervals b. Levels of phosphorus (P)

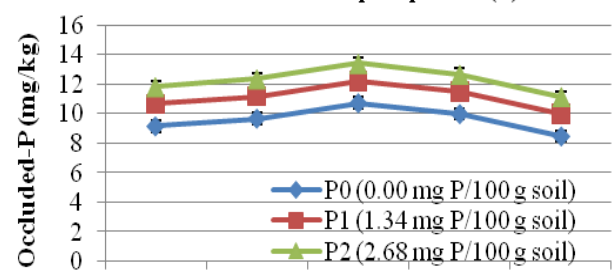

1st DAI 3rd DAI 5th DAI 7thDAI 14thDAI

Incubation intervals

d. FYM (M)

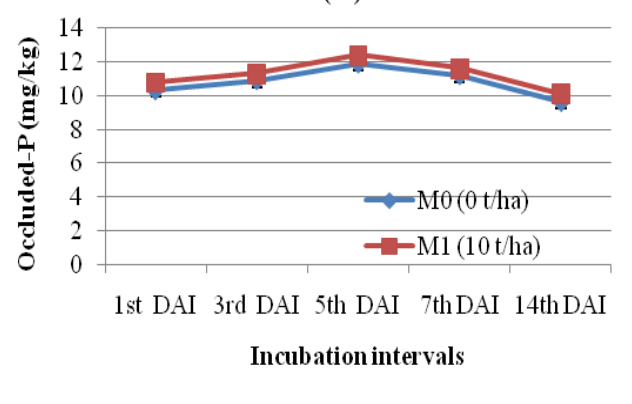


Fig.7 Effect of moisture regimes (a), FYM (d) and levels of P (b) carriers (c) on reductant-P in soil at different intervals of incubation

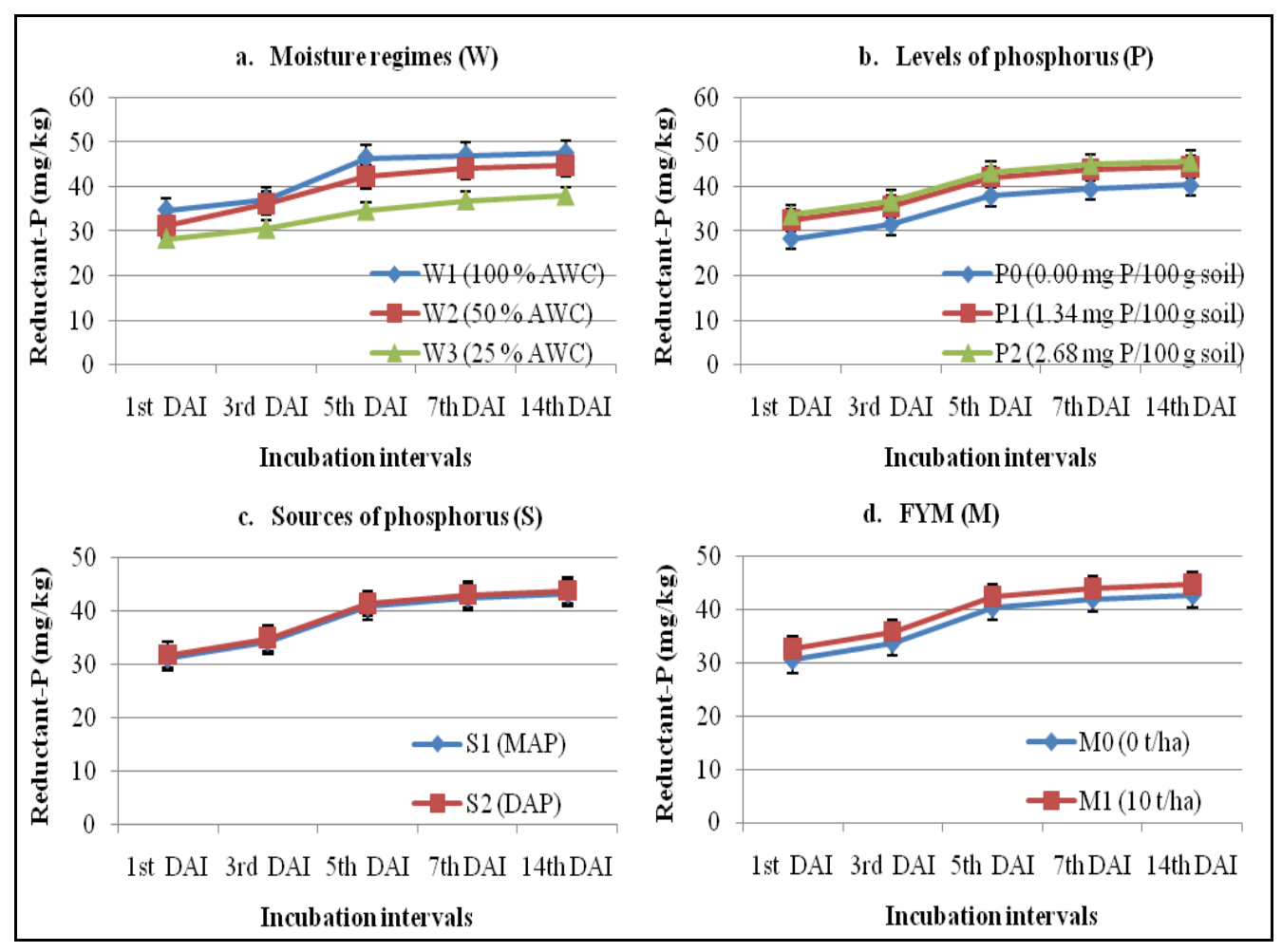

Fig.8 Effect of moisture regimes (a), FYM (d) and levels of P (b) carriers (c) on organic-P in soil at different intervals of incubation

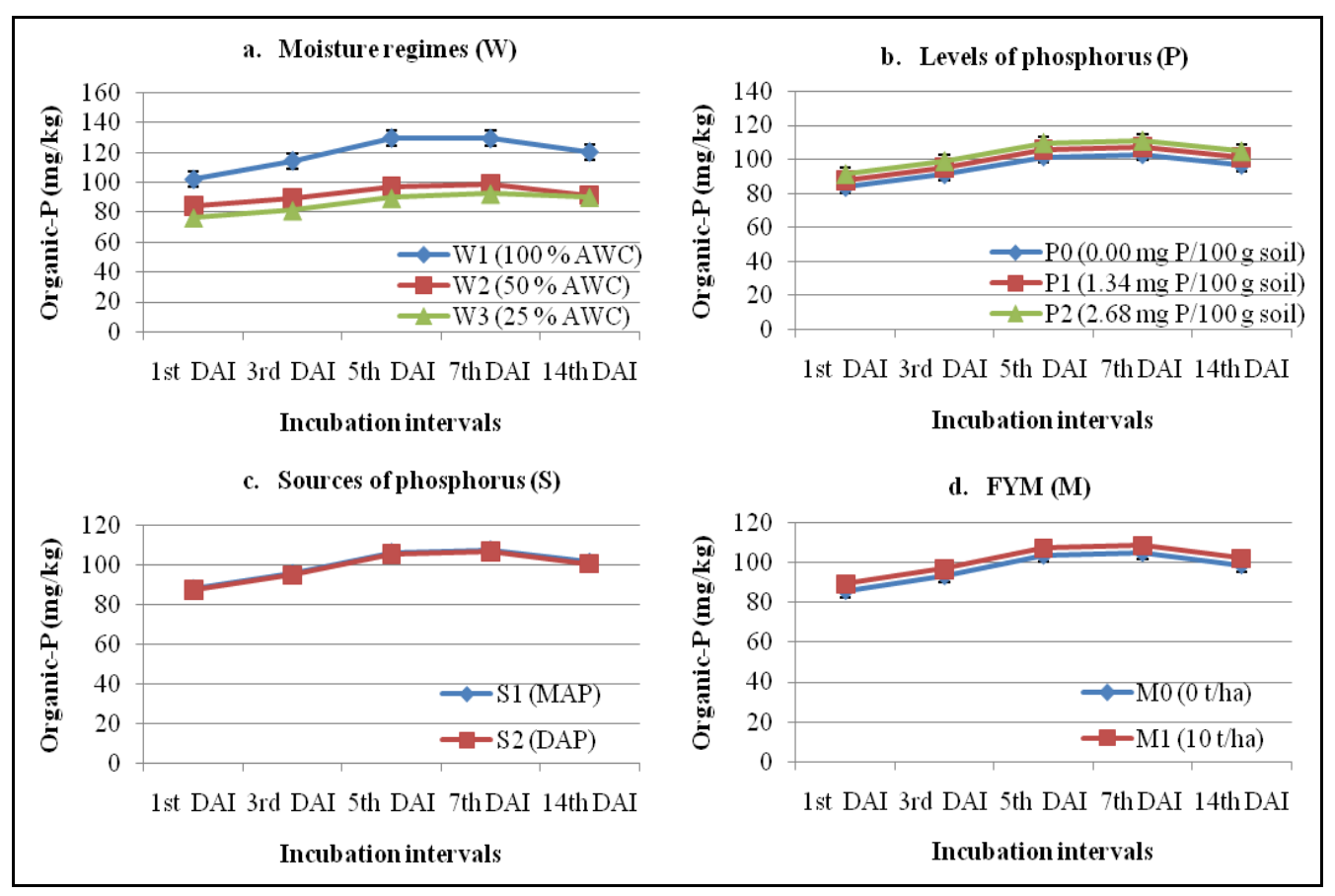


Fig.9 Effect of moisture regimes (a), FYM (d) and levels of P (b) carriers (c) on inorganic-P in soil at different intervals of incubation

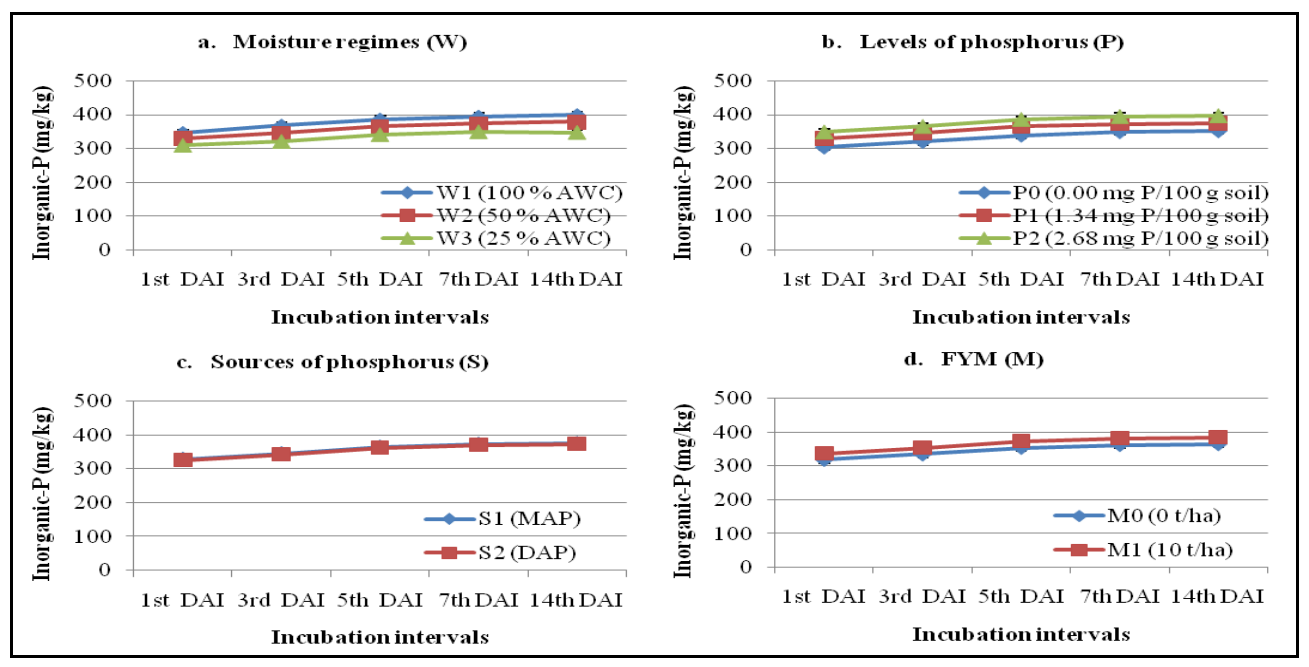

Fig.10 Effect of moisture regimes (a), FYM (d) and levels of P (b) carriers (c) on total-P in soil at different intervals of incubation

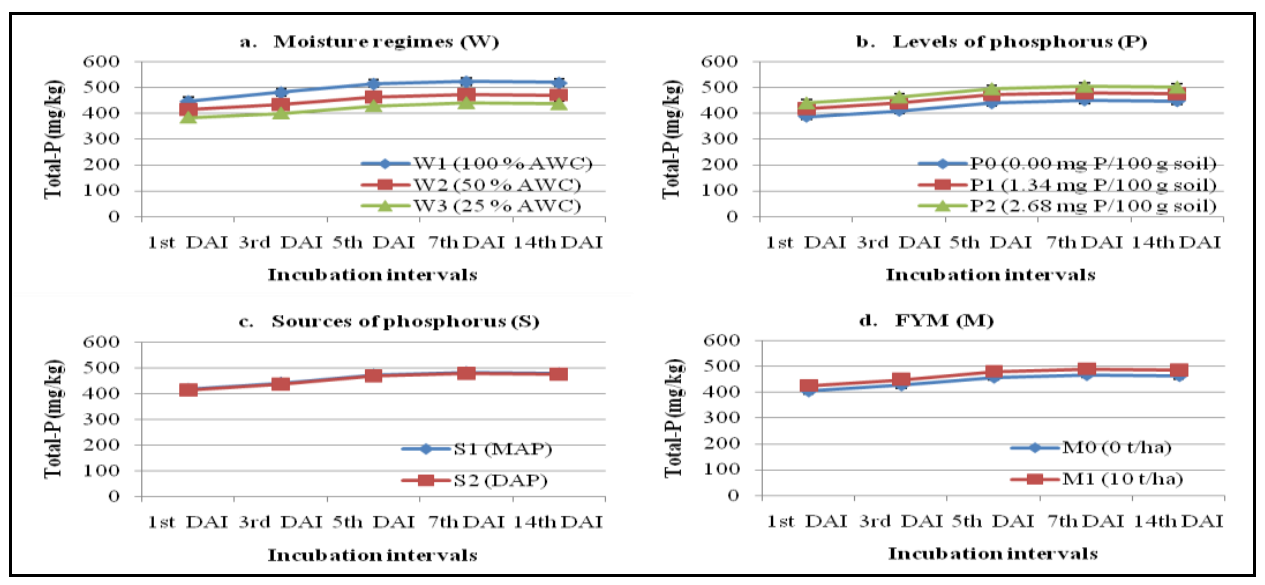

Fig.11 Effect of moisture regimes, FYM and levels of $\mathrm{P}$ carriers on per cent wise involvement of different phosphorus fraction in total-P in soil at different intervals of incubation

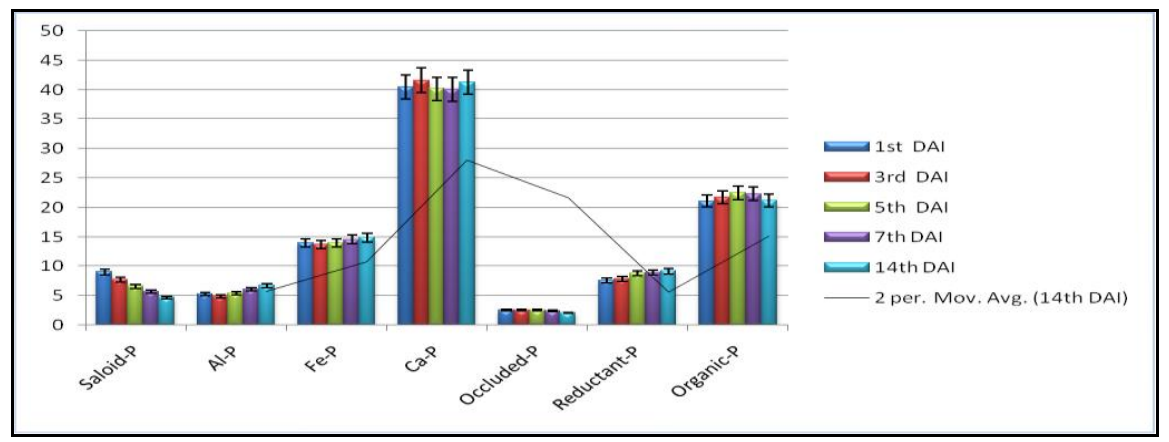


Use of FYM @ 10 t/ha $\left(\mathrm{M}_{1}\right)$ recorded significantly $(P<0.05)$ higher concentration of Inorganic-P $(395.90 \mathrm{mg} / \mathrm{kg})$ content at $1^{\text {st }}, 3^{\text {rd }}$, $5^{\text {th }}, 7^{\text {th }}$ and $14^{\text {th }}$ DAI. Whereas, treatment $\mathrm{M}_{0}$ (FYM @ 0 t/ha) recorded a lower concentration of Inorganic-P $(347.10 \mathrm{mg} / \mathrm{kg})$ during $1^{\text {st }}$ to $14^{\text {th }}$ DAI (Fig. 9(d)).

\section{Total-P (mg/kg)}

The Total-P content in soil was significantly $(P<0.05)$ influenced by moisture regimes, levels of $\mathrm{P}$ carriers and FYM was recorded at $1^{\text {st }}, 3^{\text {rd }}, 5^{\text {th }}, 7^{\text {th }}$ and $14^{\text {th }}$ DAI (Fig. 10). The overall content of Total-P was increased up to $7^{\text {th }}$ DAI, then decreasing Total-P up to $14^{\text {th }}$ DAI. A perusal of data indicated that the application of $100 \%$ available water capacity $\left(\mathrm{W}_{1}\right)$ noted a maximum Total-P (497.55 $\mathrm{mg} / \mathrm{kg}$ ) during all days after sampling (Fig. 10(a)). While, minimum Total-P content was recorded with $25 \%$ available water capacity $\left(\mathrm{W}_{3}\right)$ at $1^{\text {st }}, 3^{\text {rd }}, 5^{\text {th }}, 7^{\text {th }}$ and $14^{\text {th }}$ DAI. Total-P with application of $\mathrm{W}_{1}$ moisture regime was increased 15.84 percent, respectively over the $\mathrm{W}_{3}$ moisture regime on a mean data basis. Application of $2.68 \mathrm{mg} \mathrm{P} / 100 \mathrm{~g}$ soil resulted in a significantly $(P<0.05)$ higher Total-P over the $1.34 \mathrm{mg} \mathrm{P} / 100 \mathrm{~g}$ soil and $0.00 \mathrm{mg}$ $\mathrm{P} / 100 \mathrm{~g}$ soil during individual days after sampling. Significantly $(P<0.05)$ highest average concentration of Total-P (481.80 $\mathrm{mg} / \mathrm{kg}$ ) was obtained with an application of $2.68 \mathrm{mg} \mathrm{P} / 100 \mathrm{~g}$ soil at $1^{\text {st }}, 3^{\text {rd }}, 5^{\text {th }}, 7^{\text {th }}$ and $14^{\text {th }}$ DAI.

The behavior of Total-P content in soil was in the order of $\mathrm{P}_{0}<\mathrm{P}_{1}<\mathrm{P}_{2}$ (Fig. 10 (b)). Application of FYM @ $10 \mathrm{t} / \mathrm{ha}\left(\mathrm{M}_{1}\right)$ recorded significantly $(P<0.05)$ higher concentration of Total-P $(466.78 \mathrm{mg} / \mathrm{kg})$ at mean of $1^{\mathrm{st}}, 3^{\text {rd }}, 5^{\text {th }}$, $7^{\text {th }}$ and $14^{\text {th }}$ DAI (Fig. 10(d)). Whereas, treatment $\mathrm{M}_{0}$ (FYM @ $0 \mathrm{t} / \mathrm{ha}$ ) was recorded lower mean concentration of Total-P (444.68 $\mathrm{mg} / \mathrm{kg}$ ) during $1^{\text {st }}$ to $14^{\text {th }}$ DAI.

\section{Status of different forms of soil phosphorus} $(\%)$

The amount of $\mathrm{P}$ recovered under various fractions varied considerably depending upon the treatments given during the incubation study. All $\mathrm{P}$ fractions viz., Saloid-P, Al-P, Fe$\mathrm{P}$, Ca-P, Occluded-P, Reductant-P and Organic-P increased, when moisture level (100\% available water capacity), phosphatic fertilizers were applied at higher levels (2.68 $\mathrm{mg}$ P/100 $\mathrm{g}$ soil) either alone or in combination with organics during incubation study. The application without $\mathrm{P}$ fertilization did not influence soil $\mathrm{P}$ fractions, as under $0.00 \mathrm{mg} \mathrm{P} / 100 \mathrm{~g}$ soil treatment (Fig. 11). Ca-P with application of all treatment combinations increased 38.3 percent, over Occluded-P content as a result of mean basis. Whereas, the lowest involvement of Occluded-P content $(2.42 \%)$ in Total-P.

Phosphorus, like any other plant nutrient is present in the soil in two major components i.e. organic and inorganic. Organic $\mathrm{P}$, which is mainly confined to the surface layer, is mineralized into inorganic forms. But the plants mainly depend on inorganic $\mathrm{P}$ forms for their phosphorus requirements. Saloid-P, Al-P, Fe-P, Ca-P, Occluded-P and Reductant$\mathrm{P}$ fractions are the main source of $\mathrm{P}$ supply to the plants. The proportion of forms of phosphorus such as Ca-P, Al-P, Fe-P, Occluded-P, Reductant-P and Organic-P governs the response to applied $\mathrm{P}$ (Singh et al., 2003).

Maintenance of moisture at $25 \%$ AWC can maintain moisture at sufficient available moisture range. Due to this, phosphorus transformation in the soil is maintained, which ultimately reflected in increased $P$ availability in soil by maintaining the available moisture in the soil. Similar results are also reported by Golakiya (1988) in wheat crops. Kaloi et al., (2011) also observed a 
similar trend of availability of $\mathrm{P}$ in two different soils of Hyderabad. They observed that the release of phosphorus was increased with increasing incubation time up to 5 days after that, it was decreased with time. They revealed that the highest dose of $\mathrm{P}$ gave the maximum availability at each sampling day of the incubation period. Opala et al., (2012) studied the application of organic materials (FYM) along or in combination with inorganic P sources and stated that among the sources of organic materials the FYM was more effective.

The Saloid-P refers to the water-soluble and freely exchangeable $\mathrm{P}$ of the soil which can be reacted with the increasing moisture levels. Saloid-P content was decreased with the period of incubation in all treatments. The findings of Hanif et al., (2015) agree with the results of the present investigation. Irrespective of treatment combinations in experiments, the saloid phosphorus content of soils was found to decrease. This might be due to the transformation of soluble forms of $\mathrm{P}$ into relatively less soluble forms with time. The extent of decrease was drastic at $5^{\text {th }}$ DAI. Parallel results were reported by Singh et al., (2010). The decrease in Saloid-P beyond $3^{\text {rd }}$ to $14^{\text {th }}$ and more than that DAI may be attributed to a decrease in decomposition rate of organic manures (FYM), and therefore the quantity of organic acids and carbonic acids produced is less and the chelation of metallic ions such as $\mathrm{Fe}$ and $\mathrm{Al}$ is less. This intern enhances the conversion of Saloid-P to other forms of fractions such as Fe-P, Al-P and Ca$\mathrm{P}$. The solubilized forms of phosphates may form complexes with sesquioxides. Similar findings were observed in Manthan and Biju (1998).

Most of the water-soluble $\mathrm{P}$ added to the soil is transformed into insoluble inorganic forms depending mainly upon the chemical characteristics of soil (Mandal and Das 1970).
Also moisture has a noteworthy simulative influence on the transformation of inorganic phosphates in soil (Gupta and Kamal Nayan 1972 and Patel et al., 1992). Increased Al-P with higher rates of fertilizers $\mathrm{P}$ (Rokima and Prasad 1991) was also observed in the longterm fertilizer experiment of Palampur (Agrawal et al., 1987) and the permanent manurial experimental plots of Agricultural College, Madurai (Udaysoorian and Sree Ramulu 1991). In the case of control treatment, which did not receive $P$ fertilization, the Al-P fraction was lesser than other treatments. A higher value of Al-P content in FYM treatment as compared to control may be due to the solubilization effect of certain organic acids which are released during the FYM decomposition as reported by Patel et al., (1991). The Al-P content in soil changed widely with the continuous use of various combinations of inorganic fertilizer (Trivedi et al., 2010; Tiwari et al., 2012).

The activity of phosphorus starts immediately after the application of water and increases with time and the amount of water apply, it is generally acknowledged that concentration of $\mathrm{Fe}^{2+}$ ions increases under saturation and flooding due to the reduction of $\mathrm{Fe}^{3+}$ to $\mathrm{Fe}^{2+}$ simultaneously. The lower release of $\mathrm{P}$ at the later phase of the incubation period could probably consist of complex combination and mineral dissolution and precipitation of $\mathrm{P}$ with exchangeable cations or cations within lattice (Singh et al., 2015). Dikshit and Padihar (1988) while investigating the effect of phosphatic fertilizer on the transformation of applied $\mathrm{P}$ in a pot culture and incubation studies, found that the amount of available phosphorus and different fractions in soil increased due to phosphorus application. Subramanian and Gopalswamy (1991) obtained a significant increase in different $\mathrm{P}$ fractions by application of FYM at all the $\mathrm{P}$ levels, Hundal et al., (1991) also obtained similar results. 
Saha et al., (2000) conducted an incubation study to quantify the influence of different moisture levels and fertilizer phosphorus on the transformation of inorganic phosphates. The results showed that $\mathrm{Ca}-\mathrm{P}$ contents significantly increased even in control treatment with an incrassating level of moisture and incubation time. The results indicate that as the $\mathrm{P}$ fertilizer dose increased, the status of Ca-P also increased correspondingly at different incubation intervals. Calcium-P was found to be the dominant $\mathrm{P}$ fraction among various inorganic $\mathrm{P}$ forms present in this soil. Similar results were also reported by Singh et al., (2010). The $\mathrm{Ca}-\mathrm{P}$ was the major inorganic $\mathrm{P}$ fraction in all the treatment plots because calcareous soils are reported to have large amounts of $\mathrm{P}$ as $\mathrm{Ca}-\mathrm{P}$, irrespective of nature and kind of added fertilizer due to the more stabilized nature of calcium system under high $\mathrm{pH}$ (Jaggi 1991). Khankhane and Yadev (2000) conducted an incubation study with farmyard manure and biogas slurry added in sandy clay loam soil with or without fertilizers. The results showed that among the inorganic $\mathrm{P}$ fractions, Ca-P content in soil increased significantly with an increasing period of incubation. However, the magnitude was more in the case of farmyard manure.

The Occluded-P content increased with increased moisture levels. The findings of Hanif et al., (2015) agree with the results of the present investigation. The transformations of native and added phosphates into Occluded-P form were generally low. The application of phosphatic fertilizers increased amounts of Occluded-P. In a study (Rokima and Prasad 1991) on the transformation of $P$ added in calcareous soil at the rate of 0,50 , 75 and $100 \%$ recommended dose $\left(60 \mathrm{~kg} \mathrm{P}_{2} \mathrm{O}_{5}\right.$ $\mathrm{ha}^{-1}$ ) in combination with organic manure (FYM), it was found that most of the added $\mathrm{P}$ was transformed into Organic-P and Ca-P (40 to $45 \%$ of total) and very little to Occluded-P than other forms of Inorganic-P. The lowest concentration of Reductant-P was recorded with $25 \%$ available water capacity $\left(\mathrm{W}_{3}\right)$. Similar results were also reported by Jha and Ratan (2007) at IARI Farm, New Delhi. Subramanian and Gopalswamy (1991) obtained a significant increase in different $\mathrm{P}$ fractions by application of FYM at all the $\mathrm{P}$ levels The higher value of Total-P was evident in FYM treatment and it may be because FYM itself contains $\mathrm{P}$ which is in organic form released slowly, resulting in higher Total-P. It is in line with the findings of Venkatesh Bharadwaj et al., (1994), who reported an increase of 20.29 percent in Total$\mathrm{P}$ with FYM application.

In conclusions, this research demonstrated that the incubation study carried out available $\mathrm{P}_{2} \mathrm{O}_{5}$ content in soil was increased with the application of FYM with increasing levels of phosphorus and maintenance of moisture at $25 \%$ available water capacity as compared to without FYM. Available $\mathrm{P}_{2} \mathrm{O}_{5}$ increased up to $3^{\text {rd }}$ DAI then decreased concerning phosphorus availability, under the different forms of phosphorus, maintenance of moisture at $100 \%$ available water capacity and increased any source of inorganic phosphatic fertilizer dose in soil with FYM increased the Inorganic-P (Saloid-P, Al-P, Fe$\mathrm{P}, \mathrm{Ca}-\mathrm{P}$, Occluded-P and Reductant-P) and Total-P up to $14^{\text {th }}$ DAI in in loamy sand.

\section{References}

Agarwal S, Singh TA, Bhardwaj V (1987) Inorganic soil phosphorus fractions and available phosphorus as affected by long term fertilisation and cropping pattern in Nainital Tarai. Journal of the Indian Society of Soil Science 35:25-28

Al-Oud SS (2011) Improving phosphorus availability from phosphate rock in calcareous soils by amending with organic acid, sulfur, and/or organic 
manure. Ozean J Applied Sci 4:227-235

Bahl GS, Toor GS (2002) Influence of poultry manure on phosphorus availability and the standard phosphate requirement of crop estimated from quantity-intensity relationships in different soils. Bioresource Technol 85:317-322

Chang SC, Jackson ML (1957) Fraction of soil forms of phosphorus. Soil Science 84:133-144

Dikshit PR, Padihar SK (1988) Effect of soil moisture regime and applied phosphorus on inorganic phosphate fractions and availability of phosphorus to rice grown in a Vertisol. ORYZA 25(2):157-163

Dotaniya ML (2012) Crop residue management in rice-wheat cropping system. First Edition, Lap Lambert Academic Publisher, Germany, pp 116

Fan J, Hao MD, Wang YG (2003) Effects of rotation and fertilization on soil fertility on upland of Loess Plateau. Res. Soil Water Conserv (in Chinese) 10(1):3136

Fuentes B, de la Luz Mora M, Bolan NS, Naidu R (2008) Assessment of phosphorus bioavailability from organic wastes in soil. Developments in Soil Science, Chemical Bioavailability Terrestrial Environ 32:363-411

Golakiya BA (1988) A Systems Approach to Compromise $\mathrm{P} \times \mathrm{Z} \mathrm{n}$ Antagonism in What Growing Under Different Soil Water Conditions, Ph.D. Thesis, Gujarat Agricultural University, India, Junagadh Campus, Junagadh

Gupta ML, Kamal Nayan (1972) Transformation of soil inorganic phosphorus in red soil. Journal Indian Society of Soil Science 23:61-65

Hanif HU, Arshad M, Ali MA, Ahmed N, Qazi, IA (2015) Phytoavailability of phosphorus to Lactuca sativa in response to soil-applied $\mathrm{TiO}_{2}$ nanoparticles. Pakistan Journal of
Agricultural Science 52(1):177-182

Hesse PR (1971) A textbook of soil chemical analysis, John Murray, London, pp 255300

Hopkins BG, Ellsworth JW (2005) Phosphorus availability with alkaline/calcareous soil. Western Nutrient Management Conference. Salt Lake City, UT, USA, 6:88-93

Hundal HS, Dev G, Biswas CR (1991) Effect of green manure and kinetics of dissolved and labile $\mathrm{P}$ release in anoxic soil. Journal of the Indian Society of Soil Science 39:260-265

Ivanova R, Bojinova D, Nediaalkova K (2006) Rock phosphate solubilisation by soil bacteria. Journal of University Chemical Technology Metallurgy 41:297-302

Iyamuremye F, Dick RR (1996) Organic amendments and phosphorus sorption by soils. Adv Agron 56:139-185

Jaggi RC (1991) Inorganic phosphate fractions as related to soil properties in some representative soils of Himachal Pradesh. Journal of the Indian Society of Soil Science 39:567-568

Jakson ML (1978) Soil chemical analysis, Prentice Hall of India Private Ltd, New Delhi

Jha P, Ratan RK (2007) Mineralization of carbon and phosphorus in soil at affected by soil moisture regime and incorporation of crop residues. Journal of the Indian Society of Soil Science 55(2):218-221

Kaloi GM, Bhughio N, Panhwar RN, Junejo S, Mari AH, Bhutto MA (2011) Influence of incubation period on phosphate release in two soils of district Hyderabad. Journal of Animal and Plant Science 21(4):665-670

Karimi F, Bahmanyar MA, Shahabi M (2012) Investigation the effects of sulfur and cattle manure application on macronutrient availability in calcareous 
soil and accumulation in leaf and seed of canola. European Journal of Experim Biol 2:836-842

Khankhane PJ, Yadav BR (2000) Relative mineralization of nitrogen and phosphorus from farmyard manure, biogas slurry and sewage sludge. Journal of the Indian Society of Soil Science 48:793-797

Lai L, Hao MD, Peng LF (2003) The variation of soil phosphorus of longterm continuous cropping and management on Loess Plateau. Res Soil Water Conserv (in Chinese) 10(1):68-70

Mandal LN, Das RK (1970) Transformation of applied water-soluble phosphate in acidic lowland rice soils. Soil Science 110:263-267

Manthan CK, Biju J (1998) Influence of different fertilizer sources on phosphorus dynamics. Journal of the Indian Society of Soil Science 46(4):686-688

Marschner H (1986) Mineral Nutrition of Higher Plants. Academic Press, London

Misra SG, Ojha SK (1968) Fate of soluble phosphate applied to soils. Journal of Agricultural Science 38:837-844

Nielsen UN, Prior S, Delroy B, Walker JKM, Ellsworth DS, Powell JR (2015) Response of belowground communities to short-term phosphorus addition in a phosphorus-limited woodland. Plant and Soil 391:321-331

Opala PR, Okalebo JR, Othieno CO, Kisinyo $P$ (2012) Effect of organic and inorganic phosphorus sources on maize yields in an acid soil in Western Kenya. Nutrient Cycle of Agro-ecosystem 86(3):317-329

Patel MS, Patil RG, Zalawadia MH, Sutaria GS (1991) Effect of phosphorus, moisture and organic matter on $\mathrm{P}$ availability and its effect in a calcareous clay soil at different incubation period. Journal of the Indian Society of Soil
Science 39:316-322

Patel MS, Patil RG, Zalawadia NM, Sutaria GS (1992) Effect of phosphorus, moisture and organic matter on phosphorus availability and its fractions in a calcareous soil at different incubation periods. Journal of the Indian Society of Soil Science 4:705710

Petersen GW, Corey RB (1966) A modified Chang and Jackson procedure for routine fractionation of inorganic soil phosphates. Soil Science Society of America 30(5):563-565

Rokima J, Prasad B (1991) Integrated nutrient management-II. Transformations of applied $\mathrm{P}$ into inorganic $\mathrm{P}$ fractions in relation to its availability and uptake in calcareous soil. Journal of the Indian Society of Soil Science 39:703-709

Saha AK, Saha N, Pal SK (2000) Influence of farmyard manure and fertilizer phosphorus on transformation of inorganic phosphates and its uptake by wheat. Indian Agriculturist 44:185-191

Sanyal SK, De Datta SK (1991) Chemistry of Phosphorus Transformations in Soil. In: Stewart B.A. (eds) Advances in Soil Science. Advances in Soil Science Vol 16, Springer, New York, NY

Sardans J, Penuelas J (2007) Drought changes phosphorus and potassium accumulation patterns in an evergreen Mediterranean forest. Functional Ecology 21:191-201

Sharpley AN, Sims JT, Pierzynski GM (1994) Innovative soil phosphorus availability indices: Assessing inorganic phosphorus. In Havlin, J and Jacobsen, J (eds.) Soil Testing: Prospects for Improving Nutrient Recommendations. SSSA Special. Publication No. 40. Soil Science Society of America, Madison, USA, pp 115-142

Singh HK, Singh S, Singh DK (2015) Release Pattern of Available Phosphorus and 
Sulphur at Constant Moisture Regime in Different Soil. Journal of the Indian Society of Soil Science 63(2):228-231

Singh RP, Singh B, Dhillon NS (2010) Effect of long-term differential fertilization on distribution of inorganic $\mathrm{P}$ fractions and $P$ nutrition of wheat under maize-wheat sequence. Journal of the Indian Society of Soil Science 58(2):237-240

Singh SK, Baser BL, Shyampura RL, Narain P (2003) Phosphorus fractions and their relationship to weathering indices in Vertisols. Journal of the India Society of Soil Science 51:247-251

Subramanian KS, Gopalswamy A (1991) Effect of moisture, organic matter, phosphate and silicate on availability of silicon and phosphorus in rice soils. J of the Indian Society of Soil Science 39:99-103

Suriyagoda LDB, Ryan MH, Renton M, Lambers H (2011) Above-and belowground interactions of grass and pasture legume species when grown together under drought and low phosphorus availability. Plant and Soil 348:281-297

Tiwari HN, Singh D, Prakash V (2012) Fractions of soil phosphorus under different cropping patterns. Annals of
Plant and Soil Research 14(2):173-174

Trivedi SK, Tomar RAS, Tomar PS, Gupta N (2010) Vertical distribution of different forms of phosphorus in Alluvial soils of gird region of Madhya Pradesh. J of the Indian Society of Soil Science 58(1):8690

Udiyasoorian C, Shree Ramulu US (1991) Effect of continuous manuring and fertilizer on soil phosphorus fractions in an Alfisol under rice-rice cropping system. Madras Agricultural Journal 78:264-266

Venkatesh B, Bansal SK, Maheshwar SC (1994) Long-term effects of continuous rotational cropping and fertilization on crop yield and soil properties. $J$ of the Indian Society of Soil Science 42:220224

Verma SL (2013) Effect of compost on soil phosphorus availability (Ph.D. Thesis). School of Agriculture, Food and Wine, Waite Research Institute, the University of Adelaide, Adelaide, Australia

Zhu W, Xue J, Wang J (2004) Effects of water stress on net photosynthesis of Alnus formosana provenances and its drought tolerance. J of Soil and Water Conservation 18:170-173

\section{How to cite this article:}

Kashyap N. Patel, D. A. Patel, Vidhi K. Patel, Foram B. Patel, V. R. Patel and Pavaya, R. P. 2020. Effect of Moisture Regimes, FYM and Levels of P Carriers on Phosphorus Fractions Status of Loamy Sand in Laboratory Condition. Int.J.Curr.Microbiol.App.Sci. 9(08): 571-588. doi: https://doi.org/10.20546/ijcmas.2020.908.065 